\title{
Anti-citrullinated protein antibodies cause arthritis by cross-reactivity to joint cartilage
}

\author{
Changrong Ge, ${ }^{1}$ Dongmei Tong, ${ }^{1,2}$ Bibo Liang, ${ }^{1,2}$ Erik Lönnblom, ${ }^{1}$ Nadine Schneider, ${ }^{3}$ \\ Cecilia Hagert, ${ }^{4}$ Johan Viljanen, ${ }^{5}$ Burcu Ayoglu, ${ }^{6}$ Roma Stawikowska, ${ }^{7}$ Peter Nilsson, ${ }^{6}$ \\ Gregg B. Fields, ${ }^{7}$ Thomas Skogh, ${ }^{8}$ Alf Kastbom, ${ }^{8}$ Jan Kihlberg, ${ }^{5}$ Harald Burkhardt, ${ }^{3}$ \\ Doreen Dobritzsch, ${ }^{9}$ and Rikard Holmdahl ${ }^{1,4,10}$
}

'Section for Medical Inflammation Research, Department of Medical Biochemistry and Biophysics, Karolinska Institutet, Stockholm, Sweden. 'Department of Pathophysiology, Key Lab for Shock and Microcirculation Research of Guangdong, Southern Medical University, Guangzhou, China. ${ }^{3}$ Fraunhofer Institute for Molecular Biology and Applied Ecology IME, Project Group Translational Medicine \& Pharmacology, and Division of Rheumatology, University Hospital Frankfurt Goethe University, Frankfurt, Germany. ${ }^{4}$ Medicity Research Laboratory, University of Turku, Turku, Finland; National Doctoral Programme in Informational and Structural Biology, Turku, Finland. ${ }^{5}$ Section of Organic Chemistry, Department of Chemistry - Biomedicinskt centrum, Uppsala University, Uppsala, Sweden. ${ }^{6}$ Affinity Proteomics, Science for Life Laboratory, School of Biotechnology, KTH Royal Institute of Technology, Stockholm, Sweden. ${ }^{7}$ Department of Chemistry \& Biochemistry, Florida Atlantic University, Jupiter, Florida, USA. ${ }^{8}$ Department of Rheumatology, Department of Clinical and Experimental Medicine, Linköping University, Linköping, Sweden. ${ }^{9}$ Section of Biochemistry, Department of Chemistry Biomedicinskt centrum, Uppsala University, Uppsala, Sweden. ${ }^{10}$ Center for Medical Immunopharmacology Research, Southern Medical University, Guangzhou, China.

Today, it is known that autoimmune diseases start a long time before clinical symptoms appear. Anti-citrullinated protein antibodies (ACPAs) appear many years before the clinical onset of rheumatoid arthritis (RA). However, it is still unclear if and how ACPAs are arthritogenic. To better understand the molecular basis of pathogenicity of ACPAs, we investigated autoantibodies reactive against the $\mathrm{C} 1$ epitope of collagen type II (CII) and its citrullinated variants. We found that these antibodies are commonly occurring in RA. A mAb (ACC1) against citrullinated C1 was found to cross-react with several noncitrullinated epitopes on native $\mathrm{CII}$, causing proteoglycan depletion of cartilage and severe arthritis in mice. Structural studies by $\mathrm{X}$-ray crystallography showed that such recognition is governed by a shared structural motif "RG-TC" within all the epitopes, including electrostatic potential-controlled citrulline specificity. Overall, we have demonstrated a molecular mechanism that explains how ACPAs trigger arthritis.

Authorship note: D. Dobritzsch and R. Holmdahl are co-senior authors.

Conflict of interest: R. Holmdahl is coinventor of a patent (US 7,148,020 B2) protecting the use of collagen triplehelical epitopes for diagnostic use.

Submitted: February 27, 2017

Accepted: May 23, 2017

Published: July 6, 2017

\section{Reference information:}

JCI Insight. 2017;2(13):e93688.

https://doi.org/10.1172/jci.

insight.93688.

\section{Introduction}

Rheumatoid arthritis (RA) is a chronic, severe systemic inflammatory disorder that affects $0.5 \%-1 \%$ of the world population $(1,2)$. In addition to rheumatoid factors (RFs), approximately $70 \%$ of RA patients harbor anti-citrullinated protein antibodies (ACPAs) (3). These antibodies appear many years before the clinical onset $(4,5)$. The specificities of these antibodies are quite heterogeneous. Although autoantibodies against several citrullinated antigens (such as fibrinogen, $\alpha$-enolase, vimentin, collagen type II [CII], and fibronectin) (6) have been identified, it is still unknown what causes the onset of the inflammatory attack on the joints. There is no direct evidence that RFs or ACPAs of human origin can induce arthritis.

Evidence for pathogenic effects by ACPAs have been reported from many in vitro assays, and these results support a hypothetical concept of how ACPAs might contribute to arthritis onset in vivo. For example, the immune complexes from RA synovial fluid can induce production of cytokines by peripheral blood mononuclear cells (7). Furthermore, the immune complexes containing citrullinated fibrinogen are able to stimulate macrophages via Toll-like receptor 4 and Fc $\gamma$ receptor (8). ACPAs from RA patients were shown to activate complement via both the classical and alternative pathways (9). Autoantibodies against citrulli- 
nated vimentin can activate osteoclasts (10). It is, however, unclear whether these results are due to effects by immune complex formation $(10,11)$ or dependent on specific recognition of citrullinated epitopes on the osteoclast surface (10). Importantly, treatment with antibodies targeting B cells has proven to be effective, especially in seropositive RA patients (12).

Based on these findings, it has been assumed that autoantibodies do play a role in the triggering of joint inflammation, and three different explanations have been proposed: (a) ACPAs target citrullinated proteins on osteoclasts, enhancing bone destruction that later develops into joint inflammation (11); (b) ACPAs are potentially pathogenic but either higher levels than threshold or another glycosylation pattern are needed to create more pathogenic immune complexes $(11,13)$; or (c) the specificity of the ACPAs needs to be shifted into cross-reactivity to joint proteins $(14,15)$

We have reported that a panel of murine antibodies (denoted as ACC1, ACC3, and ACC4) against citrullinated $\mathrm{C} 1$ epitope, a major B cell epitope of CII recognized in RA, could induce as well as promote arthritis in mice (16). Although these antibodies were originally established based on their reactivity to citrullinated CII, they do, like human ACPAs, cross-react with other classical citrullinated peptides, with corresponding antibody specificities widely occurring in RA (17).

These monoclonal murine ACPAs provide a unique possibility to investigate the molecular basis of arthritogenicity. Therefore, in the present study, we set out to identify cross-reactivity of the ACC1 antibody at a molecular level. We found that increased exposure of $\alpha$ chains in structurally less stable CII regions explains its affinity for the cognate epitope in both a triple-helical context or in free $\alpha$ chains. Importantly, this mode of antibody recognition implies cross-reactivity with triple-helical epitopes with or without citrulline residues in native CII that are exposed in the joints in vivo, thereby providing a clue about the molecular determinants of ACPA pathogenicity.

\section{Results}

Antibodies to the $C 1$ epitope in $R A$. To investigate the citrulline specificity of autoantibodies against the $\mathrm{C} 1$ epitope (CII359-369: ARGLTGRPGDA), we synthesized 4 homotrimeric triple-helical peptides mimicking the native epitope on CII (Figure 1A and Table 1): the wild-type peptide C1_R-R with unmodified arginine residues, peptide C1_CIT-R citrullinated at R360, peptide C1_R-CIT citrullinated at R365, and the peptide C1_CIT-CIT with both arginines substituted by citrulline. The antibody response to these peptides was analyzed in a Swedish RA cohort (TIRA2, 504 cases, 285 controls). Antibodies against each of the 4 triple-helical C1 peptides were found to be increased in RA patients compared with healthy controls $(P<$ 0.001 for all, Figure 1B and Table 1). Approximately $30 \%$ of the RA patients had similar antibody titers for the peptides C1_CIT-CIT and C1_CIT-R, indicating that the citrulline at the first position (Cit360) in the $\mathrm{C} 1$ epitope is predominant (Table 1 ).

CII can maintain an overall compact triple-helical structure in the cartilage matrix, but it is also likely that the triple-helical structure could be dynamic, leading to local flexibility of $\alpha$ chains, which allows interactions with other matrix molecules, such as integrins and enzymes (18). To explore this possibility, a set of 4 cyclic $\mathrm{C} 1 \alpha$ chain variants was synthesized and tested for antibody binding (Figure $1 \mathrm{~A}$ and Table 1$)$. The $\alpha$ chain peptides were also more frequently recognized in RA patients $(P$ $<0.001$ for all, Figure 1C and Table 1). In accordance with the binding to triple-helical peptides, the highest frequency of positive results was observed for antibodies recognizing the first citrulline at position 360 (sensitivity of $51 \%$ for C1-CIT360), whereas the peptide containing citrulline at position 365 was less frequently recognized (sensitivity of 19\% for C1-CIT365).

The question arises as to whether the antibodies against the triple-helical epitopes indeed bind the epitope when the $\alpha$ chain bulges out from the triple-helical structure, leading to cross-reactivity with the $\alpha$ chain in cyclic form. Hence, we analyzed the correlation between the occurrences of these antibodies. As shown in Figure 2A, approximately $10 \%$ of RA patients responded to unmodified C1 epitope regardless of triple-helical conformation, and half of these patients also reacted to both triple-helical (C1_R-R) and $\alpha$ chain C1 variants (C1-R360 or C1-R365). Similarly, about 30\% of RA patients reacted to both the triple-helical peptide C1_CIT-R and the $\alpha$ chain peptide C1-CIT360, and 21\% of RA patients only respond to the $\alpha$ chain C1-CIT360, whereas almost none of the patients reacted specifically to the triple-helical C1-CIT-R peptide (Figure 2B). The same relation was also found for the less prominent C1-CIT365 peptide (Figure 2B). Taken together, this suggests that the $\alpha$ chain in the triple-helical epitope could mimic a free $\alpha$ chain, i.e., most likely due to a dynamic flexibility. 
A

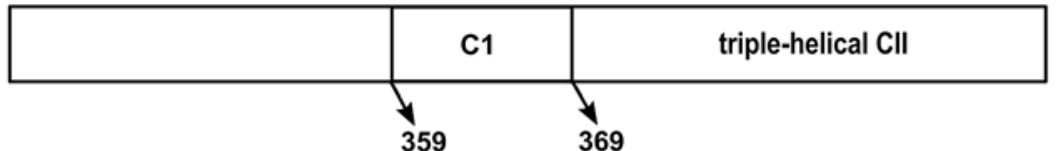

(GPO)5 - GDOGROGEOGLOGARGLTGROGDA - (GPO) 5 - G

(GPO)5 - GDOGROGEOGLOGARGLTGROGDA - (GPO) 5 - G-K-K-biotin (triple)

(GPO) 5 - GDOGROGEOGLOGARGLTGROGDA - (GPO) 5 - G-

biotin-Ahx-CEPGLPGARGLTGRPGCA (cyclic)

biotin-Ahx-CGARGLTGRPGDAGPQCA (cyclic)
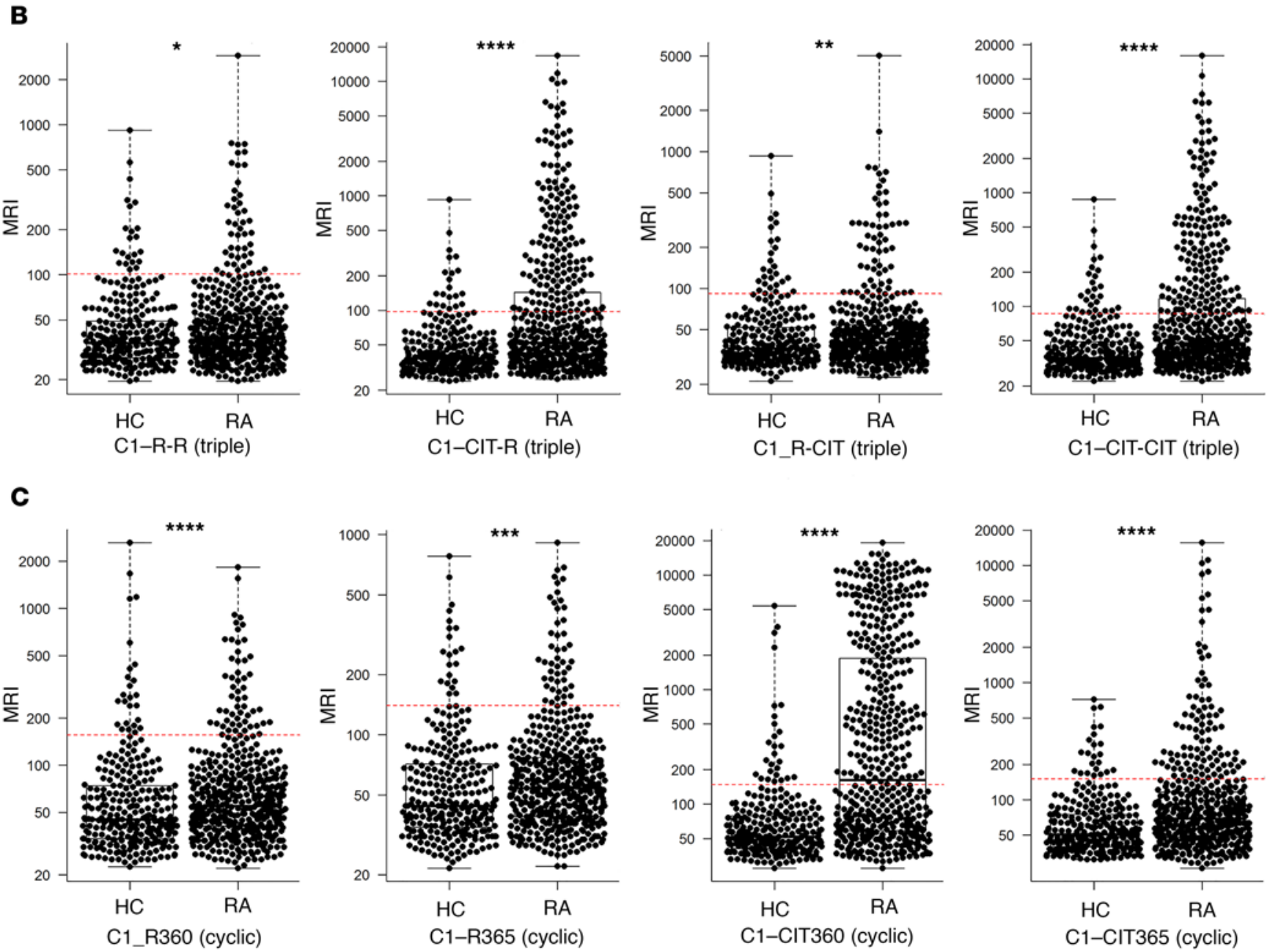

Figure 1. Anti-C1 epitope antibody responses in RA. (A) The sequence of synthetic C1 epitope (CII259-269) variants. The lysine knot placed at the C terminus establishes the requisite register for triple-helix formation with the identical peptide strands, whereas two cysteine residues were added to a single $\alpha$ chain to achieve cyclization via disulfide bonds. Biotin was added to all peptides, as indicated for detection in Luminex assay. The corresponding citrullinated peptides have the same sequence, aside from substitution of arginine with citrulline residues at the given position, i.e., 360 and 365 . 0 , (2S,4R)-4-hydroxyproline; Ahx, aminocaproic acid. (B and C) IgG response of the TIRA2 cohort (504 RA patients and 285 healthy controls) to peptides containing variants of the triple-helical C1 epitope (B) or to the cyclic $\alpha$ chain of the C1 epitope (C). All biotinylated peptides were captured on beads via recognition of NeutrAvidin, which was immobilized on the beads through amine coupling. Human serum samples were diluted 1:100 (v/v) in assay buffer and incubated for 60 minutes at room temperature on a shaker for preadsorption of unspecific antibodies. Then, the serum samples were transferred to a 384-well plate containing peptide-coated beads. After incubation at room temperature on a shaker for 75 minutes, all beads were washed with PBS-T and resuspended in a solution containing the secondary anti-human IgC Fcy-PE. After 40 minutes of incubation, the beads were washed with PBS-T and then measured. The median fluorescence intensity (MFI) was used to quantify the interaction of serum antibody with the given peptides. The red line indicates the cutoff value for positivity as described in the Methods. Mann-Whitney $U$ test was used to calculate $P$ values for differences between groups $\left({ }^{*} P<0.05,{ }^{* *} P<0.01,{ }^{* *} P<0.001,{ }^{* * *} P<0.0001\right)$. The red line indicates the cutoff (median $+5 \times$ median absolute deviation [MAD]) based on healthy controls. MFI, median fluorescence intensity; RA, rheumatoid arthritis; HC, healthy controls; RT, room temperature. 
Table 1. Diagnostic performance of Luminex immunoassay using peptide variants of C1 epitope of CII in the TIRA2 cohort

\begin{tabular}{|c|c|c|c|c|}
\hline Name & Sequence & Sensitivity & Specificity & $P$ value \\
\hline C1_R-R & $(\mathrm{GPO})_{5}$-GDOGROGEOGLOGARGLTGROGDA-(GPO) & $9 \%$ & $92 \%$ & 0.02 \\
\hline C1_CIT-R & $(G P O)_{5}$-GDOGROGEOGLOGAXGLTGROGDA-(GPO) & $31 \%$ & $93 \%$ & $3.03 \times 10^{-17}$ \\
\hline C1_R-CIT & $(G P O)_{5}-G D O G R O G E O G L O G A R G L T G X O G D A-(G P O)_{5}$ & $11 \%$ & $91 \%$ & $3.92 \times 10^{-03}$ \\
\hline C1_CIT-CIT & $(G P O)_{5}-G D O G R O G E O G L O G A X G L T G X O G D A-(G P O)_{5}$ & $30 \%$ & $91 \%$ & $8.93 \times 10^{-17}$ \\
\hline C1-R360 & CEPGLPGARGLTGRPGCA & $11 \%$ & $91 \%$ & $9.73 \times 10^{-05}$ \\
\hline C1-R365 & CGARGLTGRPGDAGPQCA & $9 \%$ & $93 \%$ & $2.90 \times 10^{-04}$ \\
\hline C1-CIT360 & CEPGLPGAXGLTGRPGCA & $51 \%$ & $91 \%$ & $1.67 \times 10^{-36}$ \\
\hline C1-CIT365 & CGARGLTGXPGDAGPQCA & $19 \%$ & $92 \%$ & $3.68 \times 10^{-11}$ \\
\hline CEP1 & CKIHAXEIFDSXGNPTVEC & $57 \%$ & $92 \%$ & $3.90 \times 10^{-49}$ \\
\hline CCP1 & HQCHQESTXGRSRGRCGRSGS & $56 \%$ & $94 \%$ & $9.69 \times 10^{-45}$ \\
\hline
\end{tabular}

The cutoff for calculation of sensitivity and specificity is computed using the median $+5 \times$ median absolute deviation of the healthy control group. Biotin is attached to the $\mathrm{N}$-terminal of all peptides. $P$ values are calculated by nonparametric Mann-Whitney $U$ test. ${ }^{A} R$ heumatoid arthritis $=504$ and heathy control = 285. 0 , hydroxylproline; $\mathrm{X}$, citrulline.

In addition, we also compared the correlation of antibody reactivity against the most prominent $\mathrm{C} 1$ peptide, C1-CIT360, and the well-known citrullinated epitope CEP1 (Figure 2B). As with most ACPAs, the antibodies against citrullinated $\mathrm{C} 1$ epitope also show a high degree of cross-reactivity with citrullinated peptides derived from different proteins, here exemplified by a partial cross-reactivity to enolase peptide CEP1. The frequency of antibody response specific for the triple-helical structure, without cross-reacting with $\alpha$ chains, was $3 \%$, but this result was still substantial (Figure $2 \mathrm{~A}$ ). Despite their infrequency, these antibodies could be of importance, as they are known to bind to cartilage and initiate arthritis in mice (19).

In summary, the above data show that ACPAs recognizing triple-helical CII most likely target the molecule when the $\alpha$ chain is not in strict triple-helical conformation but is more flexible.

Cross-reactivity of autoantibodies against citrullinated C1 epitopes. We have previously prepared a panel of $\mathrm{mAbs}$ to the citrullinated $\mathrm{C} 1$ epitope and demonstrated their pathogenicity (16). To further reveal the specificity of 3 of these mAbs (ACC1, ACC3, and ACC4) and possibly uncover previously concealed epitopes, a set of 54 cyclic peptides, based on the entire human CII sequence in which arginine is substituted by citrulline, together with the corresponding set of unmodified counterpart peptides, was synthesized and used in Luminex immunoassays (Supplemental Table 1; supplemental material available online with this article; https://doi.org/10.1172/jci.insight.93688DS1). Both ACC1 and ACC3 cross-reacted with several of the citrullinated or unmodified cyclic CII peptides to different extents (i.e., affinity). In agreement with our previous observation, ACC1 showed stronger binding affinity to C1-CIT365 than C1-CIT360 (Figure 3A and Supplemental Figure 1A). However, since the C1-CIT360 is more frequently recognized by RA sera than C1-CIT365, we performed a solid-phase inhibition assay (Supplemental Figure 1B), showing that the binding of human RA sera to the epitope C1-CIT360 was reduced by preincubation with ACC1. Similarly, the nonarthritogenic mAb GB8 (16), specifically targeting denatured CII, showed broad-spectrum responses. In contrast, ACC4 showed specific binding to only the citrullinated C1 peptides C1_CIT-R and C1_CIT360, which is in good agreement with our previous report (16). As expected, the triple-helix-specific antibody CIIC1 (20) showed specific binding to the unmodified triple-helical C1 epitope.

We further assessed the cross-reactivity of ACC1 with triple-helical peptides of CII by surface plasmon resonance (SPR). We screened a set of overlapping triple-helical peptides (each representing 8 successive G-X-Y motifs) based on the entire triple-helical region of human collagen II (Supplemental Table 1). Several triple-helical peptides were recognized by ACC1 (Figure 3B and Supplemental Table 2). The data showed ACC1 binding to the C1 epitope citrullinated at R365 (C1-T-CIT365), which is in good agreement with our previous result (16). Furthermore, the major CII epitope F4 (F4-T-CIT933) recognized by ACPAs in RA (14) also gave a positive signal. Considering possible triple-helicity defects in synthetic peptides, we further measured the binding of ACC1 against a panel of physiologically assembled recombinant triple-helical CII peptides (21) and confirmed the same positive epitopes (Supplemental Figure 1C). Importantly, the same epitopes were revealed in both the $\alpha$ chain peptide library as well as in the synthetic and recombinant triple-helical peptide library, indicating that ACC1 binds to a shared epitope, most likely to a flexed and dynamic $\alpha$ chain protruding from triple-helical CII. 

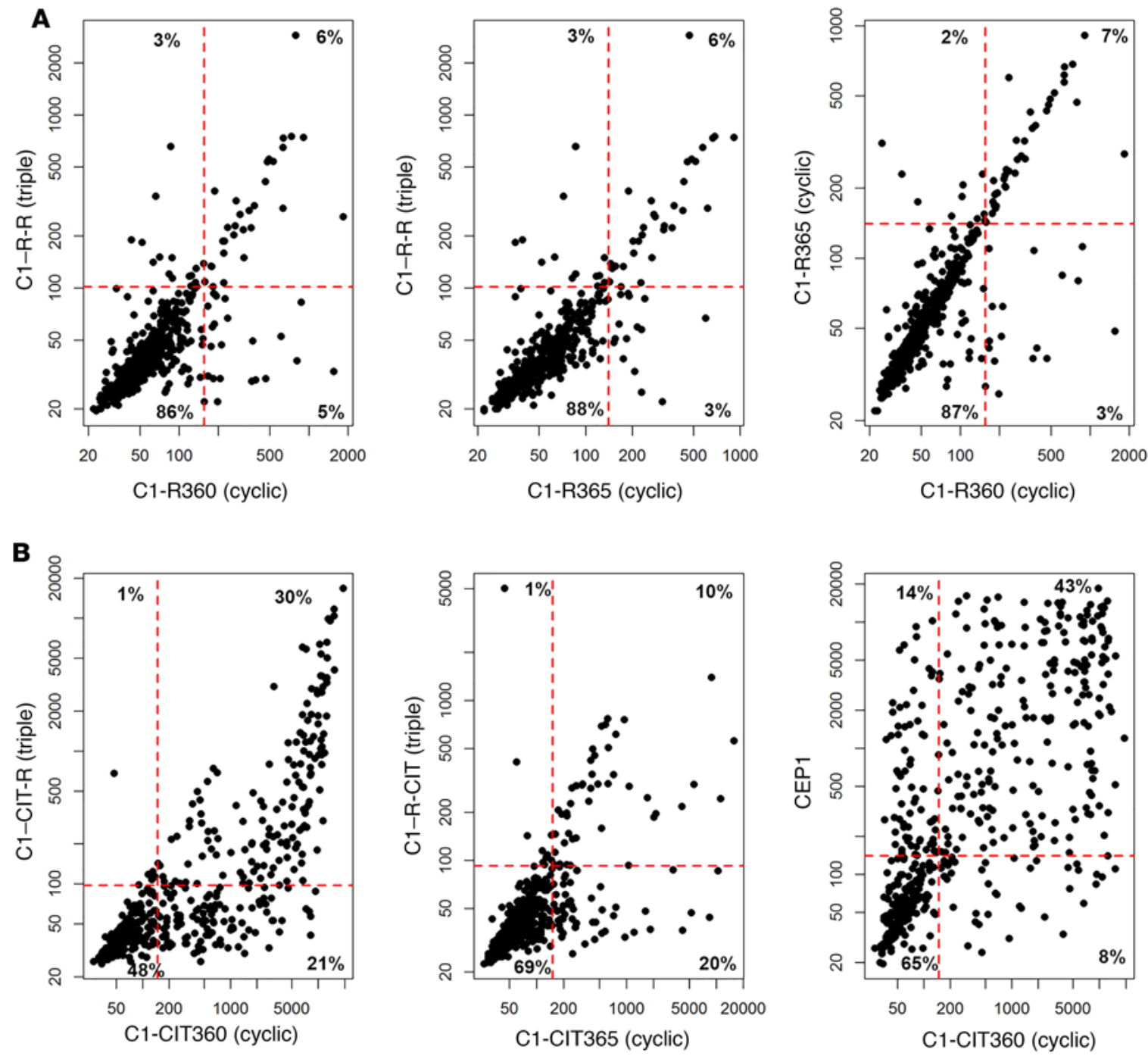

Figure 2. Correlation of antibody responses against different C1 epitope variants in RA. (A) Pairwise comparison was performed between cyclic unmodified C1 peptides (C1-R360 and C1-R365) and the triple-helical peptide containing the unmodified C1 epitope (C1_R-R). (B) Pairwise comparisons were performed between the citrullinated cyclic C1 peptides (C1-CIT360 and C1-CIT365) and the prominent triple-helical C1 peptide (C1_CIT-R). Comparison was also performed between the citrullinated cyclic C1-CIT360 and classical CEP1 peptide. A total of 504 RA patients from TIRA2 cohort was used in the analysis. MFI, median fluorescence intensity; RA, rheumatoid arthritis. The red line indicates the cutoff (median $+5 \times$ median absolute deviation [MAD]) based on healthy controls.

Characterization of peptides binding to ACC1. To gain more detailed insights into the interaction between ACC1 and its triple-helical CII epitopes, we investigated the direct binding of 9 triple-helical peptides to ACC1 by SPR. As shown in Table 2, the noncitrullinated triple-helical peptides, including CII121-144, CII241-264, CII571-591, CII916-939 (F4 epitope), and CII931-954, as well as the citrullinated triple-helical epitopes C1 (C1-T-CIT365) and F4 (F4-T-CIT933) showed considerable binding. The peptide CII571-591 showed the highest affinity for ACC1 with a $\mathrm{K}_{\mathrm{D}}$ of $4.6 \times 10^{-9} \mathrm{M}$ and a slow off-rate $\left(\mathrm{K}_{\mathrm{d} 1}=7.5 \times 10^{-3} \mathrm{~s}^{-1}\right.$, and $\mathrm{K}_{\mathrm{d} 2}=3.6 \times$ $10^{-3} \mathrm{~s}^{-1}$ ). It is noteworthy that the heterogenous binding curves can only be fitted by a two-state binding mode, implying that binding occurs with a conformational change of the antibody in a dynamic way.

$A C P A$ characteristics of ACC1. We have shown that ACC1 binds to flexible triple-helical CII determinants as well as many citrullinated CII peptides but did not further explore its reactivity toward other types of citrullinated proteins/peptides. Therefore, we analyzed the binding of ACC1 to CCP1 and an undisclosed peptide mixture from the Immunoscan CCPlus Kit (Euro Diagnostica). Clearly, ACC1 recognized both CCP1 and peptides of the Immunoscan CCPlus Kit (Figure 3, C and D), confirming that ACC1 mimics a classical ACPA in RA. 
A
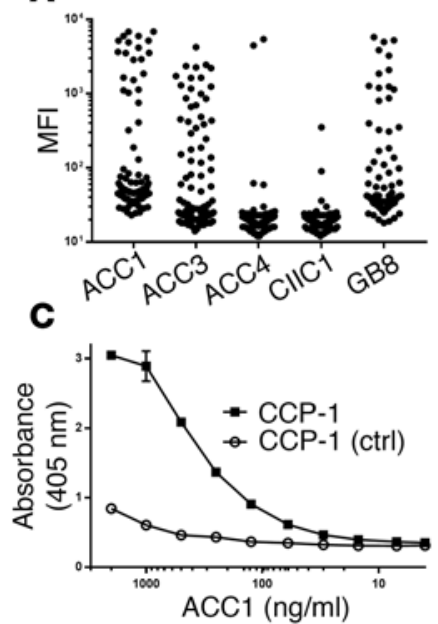

B
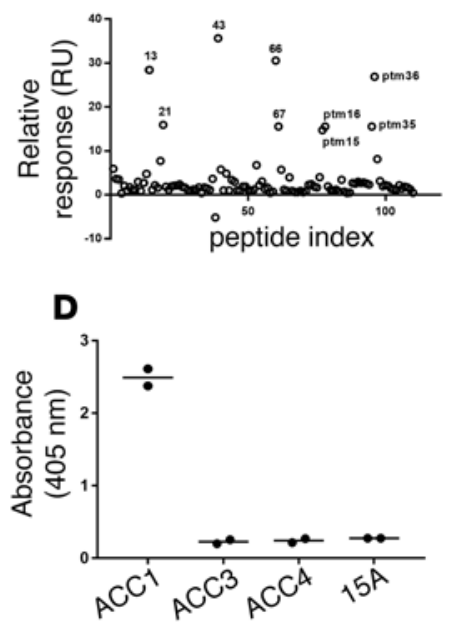

Figure 3. Characterization of the cross-reactivity of ACC1. (A) Responses of ACC1, ACC3, ACC4, CIIC1, and CB8 toward cyclic CII peptides and triple-helical CII peptides determined in the Luminex assay (Supplemental Table 2). A total of 127 peptides was used in the assay, and each dot represents a unique peptide. The median fluorescence intensity (MFI) was used to quantify the interaction of antibody with given peptides. (B) Reactivity of ACC1 toward triple-helical CII peptides (107 triple-helical peptides from Supplemental Table 1) measured by surface plasmon resonance (SPR). Sensograms were processed using an automatic correction for nonspecific bulk-refractive index effects. Data processing and analysis were performed using Biacore T200 evaluation software in a heterogenous binding model (GE Healthcare). 13, CII121-144; 21, CII241-264; 43, CII571-591; 66, CII916-939; 67, CII931-954; ptm15/ptm16, C1-T-CIT365; ptm35/36, F4-T-CIT933. (C) Preference of ACC1 for citrullinated CCP1 compared with the corresponding unmodified CCP1. Data are representative of results from triplicate assays. (D) Reactivity of ACC1, ACC3, ACC4, and 15A toward peptides from the CCP2 kit. The peptides from the Immunoscan CCPlus Kit (Euro Diagnostica) were used to measure the binding of ACC1, ACC3, ACC4, and $15 \mathrm{~A}$ (COMP-specific antibody). The absorbance value at $405 \mathrm{~nm}$ was used to quantify the binding capacity. Data are representative of results from two assays performed using duplicate technical replicates. SPR, surface plasmon resonance; CII, collagen type II.

ACC1 binds to cartilage and induces arthritis. To evaluate the pathogenicity of ACC1 in mice, we compared its arthritogenicity with that of the arthritogenic anti-CII antibody M2139 in B10.Q mice. In agreement with our previous report (16), ACC1 alone induces as severe arthritis as the positive control M2139, and a combination of both did not increase the severity further (Figure 4, A and B). In addition, the arthritis appeared even before the LPS boost given at day 5 that is normally used to enhance development of arthritis. Histopathologic examinations of joints confirmed that all 3 groups of mice had severe synovitis, as shown by hyperplasia, inflammatory cell infiltration, angiogenesis, and pannus formation (Supplemental Figure 2).

To test whether the ACC1 antibody binds triple-helical CII expressed physiologically, we showed by immunohistochemistry that it bound strongly to mouse cartilage both in vitro and in vivo (Figure 4, C and D). In addition, the binding of ACC1 led to depletion of proteoglycan, which is most likely the first step toward the development of arthritis. To investigate whether there are also targets for ACC1 present in RA cartilage, we performed human cartilage staining with ACC1 using the anti-CII antibody CIIC1 as a positive control. As shown in Figure 4, E and F, massive staining was observed for ACC1 in cartilage derived from two different RA patients, while the positive control CIIC1 showed weaker but substantial binding. Importantly, this shows that ACC1 is specific for cartilage. Together, these data further support that ACC1 reacts strongly to epitopes exposed in cartilage.

Overall structure of $\mathrm{ACC1}_{\mathrm{Fab}}$-peptide complexes. To identify the structural determinants governing the citrulline specificity, epitope-conformation specificity, and cross-reactivity of ACC1, we cocrystallized the Fab fragment with 4 different CII peptides, C1-CIT365-L, C1-CIT365-T, CII583-591, and CII616-639CIT (Supplemental Table 3), and determined the structure of the respective complexes to resolutions of 2.7-3.1 A by molecular replacement. C1-CIT365-L is a linear, single-chain form of the C1 epitope, while C1-CIT365-T is the counterpart with triple-helical structure. CII583-591 contains a part of the noncitrullinated epitope CII571-591 that was shown to be the strongest binder to ACC1 (c.f., above). CII616-639-CIT, harboring yet another citrullinated CII epitope, showed strong affinity in ELISA, though it showed no positive signal in our SPR screening assay. The full data collection and refinement statistics are summarized in Table 3. The 4 immunoglobulin domains comprising the Fab fragment are each stabilized by an intramolecular disulphide bond (VH:22-H:98, CH:143-H:198, VL:23-L:92, and CL:138-L:198) (Figure 5A). The low root-mean-square deviations (RMSDs) obtained for pairwise superposition of the respective individual Ig domains reveal that the structures of the $\mathrm{ACC} 1_{\mathrm{Fab}}$ copies within the asymmetric unit or of the various

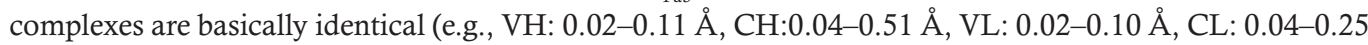
$\AA$ for $\mathrm{ACC}_{\mathrm{Fab}}-\mathrm{CII616-639-CIT} \mathrm{complex).} \mathrm{The} \mathrm{peptide} \mathrm{sequences} \mathrm{are} \mathrm{only} \mathrm{partially} \mathrm{visible} \mathrm{in} \mathrm{electron} \mathrm{den-}$ sity, as both termini protrude from the Fab binding groove and are disordered (Supplemental Figure 3A). The maximal extent of their modeling is indicated in Supplemental Table 3. All 4 peptides are bound as 
Table 2. Kinetic and equilibrium dissociation constants determined by surface plasmon resonance for the interactions of ACC1 and a set of triple-helical peptides of CII

\begin{tabular}{lcccccccc}
\hline Name & Peptide nomenclature & $\begin{array}{c}\mathbf{K}_{\mathrm{a}} \\
\left(\mathbf{M}^{-1} \cdot \mathbf{s}^{-1}\right)\end{array}$ & $\begin{array}{c}\mathbf{K}_{\mathrm{a} 1} \\
\left(\mathbf{M}^{-1} \cdot \mathbf{s}^{-1}\right)\end{array}$ & $\begin{array}{c}\mathbf{K}_{\mathrm{a} 2} \\
\left(\mathbf{M}^{-1} \cdot \mathbf{s}^{-1}\right)\end{array}$ & $\begin{array}{c}\mathbf{K}_{\mathrm{d}} \\
\left(\mathbf{s}^{-1}\right)\end{array}$ & $\begin{array}{c}\mathbf{K}_{\mathrm{d} 1} \\
\left(\mathbf{s}^{-1}\right)\end{array}$ & $\begin{array}{c}\mathbf{K}_{\mathrm{d} 2} \\
\left(\mathbf{s}^{-1}\right)\end{array}$ & $\begin{array}{c}\mathbf{K}_{\mathbf{v}} \\
(\mathbf{M})\end{array}$ \\
CII121-144 & CII_LT_E41_R-R-R & - & $8.3 \times 10^{4}$ & $6.9 \times 10^{-3}$ & - & $5.9 \times 10^{-2}$ & $4.9 \times 10^{-3}$ & $2.9 \times 10^{-7}$ \\
CII241-264 & CII_LT_252 & - & $1.1 \times 10^{5}$ & $3.0 \times 10^{-3}$ & - & $2.1 \times 10^{-1}$ & $6.8 \times 10^{-3}$ & $1.4 \times 10^{-6}$ \\
CII571-591 & CII_LT_582 & - & $2.1 \times 10^{5}$ & $2.5 \times 10^{-2}$ & - & $7.5 \times 10^{-3}$ & $3.6 \times 10^{-3}$ & $4.6 \times 10^{-9}$ \\
CII916-939 & CII_LT_F4_R-R & - & $1.3 \times 10^{5}$ & $3.2 \times 10^{-3}$ & - & $4.6 \times 10^{-2}$ & $2.2 \times 10^{-3}$ & $1.4 \times 10^{-7}$ \\
CII931-954 & CII_LT_(F4)_(R)-R & - & $1.2 \times 10^{5}$ & $4.3 \times 10^{-3}$ & - & $9.8 \times 10^{-2}$ & $4.6 \times 10^{-3}$ & $4.3 \times 10^{-7}$ \\
C1-T-CIT365 & CII_LT_C1_R-CIT & - & $4.9 \times 10^{3}$ & $3.9 \times 10^{-3}$ & - & $1.4 \times 10^{-1}$ & $7.0 \times 10^{-3}$ & $1.8 \times 10^{-5}$ \\
F4-T-CIT933 & CII_LT_F4_R-CIT & - & $8.9 \times 10^{3}$ & $3.4 \times 10^{-3}$ & - & $9.5 \times 10^{-2}$ & $3.9 \times 10^{-3}$ & $5.7 \times 10^{-6}$ \\
F4-T-CIT933 & CII_LT_F4_CIT-CIT & - & $1.7 \times 10^{4}$ & $3.8 \times 10^{-3}$ & - & $1.0 \times 10^{-3}$ & $3.8 \times 10^{-3}$ & $4.2 \times 10^{-6}$
\end{tabular}

Peptides were injected over the immobilized ACC1. In all cases, the heterogeneous binding curves were fitted using a two-state reaction model with Biacore T200 Evaluation Software.

single chains in a remarkably similar S-shaped manner by the $\mathrm{ACC}_{\mathrm{Fab}}$ paratope in the crystal structures (Figure 5, B-D). Approximately 2.5\% (250-300 $\left.\AA^{2}\right)$ of the solvent-accessible surface area (SASA) of both chains and $700-760 \AA^{2}$ of the respective peptides (ca. $55 \%$ of SASA) are buried upon complex formation.

The superposition of all $\mathrm{ACC1}_{\mathrm{Fab}}$-peptide complexes (Figure 5, B-D, and Supplemental Figure 3B) highlights that binding of the 3 distinct epitopes engages the same paratope and does not require pronounced changes in complementarity-determining region (CDR) loop conformation: those of loops $\mathrm{H} 1, \mathrm{H} 2, \mathrm{H} 3$, and L2 are basically identical, whereas the tips of the L1 and L3 loops shift position only by up to $2 \AA$ to adjust to a sequence variation upstream of the conserved motif (Cit-Hyp in C1-CIT365-T and C1-CIT365-L, corresponding to Pro-Ile in CII583-591 and Pro-Hyp in CII616-639) (Supplemental Figure 3C).

Conserved motif-mediated interactions in the $A C C 1_{F a b}$-peptide complexes. The $\mathrm{L} 1$ and $\mathrm{H} 1$ loops provide most of the SASA upon complex formation. $\mathrm{H} 1$ contacts the $\mathrm{N}$-terminal part of the peptides up to the conserved arginine, while the H3 loop residues 101-104 play a crucial role in coordinating the entire RG-TG motif (Figure 5E). L1 binds to the motif threonine and the third and fourth residue thereafter, and L3 binds to the two residues in between. The contributions of $\mathrm{H} 2$ and $\mathrm{L} 2$ are almost negligible, though $\mathrm{H} 2$ plays, via residue heavy-chain R52 (hR52), a more prominent role for binding of the citrullinated peptides (see below).

The residues light chain F40 (IF40), 1F50 and 1Y53, belonging to framework region FRL2, are also involved in shaping the peptide-binding paratope. Moreover, they provide a large proportion of the $\mathrm{ACC}_{\mathrm{Fab}}$ hydrophobic interaction surface for peptide binding, especially for the motif threonine and the preceding nonconserved residue. Other prominent hydrophobic contacts with the peptide are made by hW33 and hL101.

Peptide binding by the $\mathrm{ACC}_{\mathrm{Fab}}$, however, is primarily achieved via polar contacts (Figure 5E and Supplemental Figure 4). There are several key hydrogen bond interactions that are conserved in all complexes, despite the differences in sequence of the bound peptides (Figure 6; Supplemental Figure 5; and Supplemental Figure 6, C and D). These include the peptide conformation-stabilizing carbonyl oxygen-amide nitrogen interaction between the glycines of the conserved RG-TG motif. The corresponding interaction between the first of these glycines and that of the preceding amino acid triplet is also observed in about half of the $\mathrm{ACC} 1_{\mathrm{Fab}}$-peptide complex copies. The motif threonine is clearly the interaction hot spot: its side chain fits perfectly in the paratope, and its hydroxyl is placed such that it can form 3 hydrogen bonds simultaneously, with the backbone amides of $\mathrm{hD} 104$ and $\mathrm{hF} 103$ and the side chain of $\mathrm{hD} 104$. Alternatively, the carbonyl oxygen of $\mathrm{hL} 101$ can replace the latter as interaction partner. The shape complementarity and multitude of possible polar interactions of the threonine side chain explain its conservation in all peptide sequences recognized by ACC1. The remaining hydrogen bond network with the Fab is dominated by interactions that involve the peptide backbone (Figure 6; Supplemental Figure 5; and Supplemental Figure 6, A-D), which may be the reason for the enhanced cross-reactivity of ACC1.

A higher peak of electron density near the guanidinium group of the motif arginine in all $\mathrm{ACC}_{\mathrm{Fab}}$ complexes indicates the presence of a counter ion (Figure 5E); however, in most cases, it could not be identified and hence was not modeled. For the $\mathrm{ACC}_{\mathrm{Fab}}$-CII616-639 complex, for which the observed density peak was strongest and suggestive of a chemical entity, modeling and refinement of the counter ion as 
A

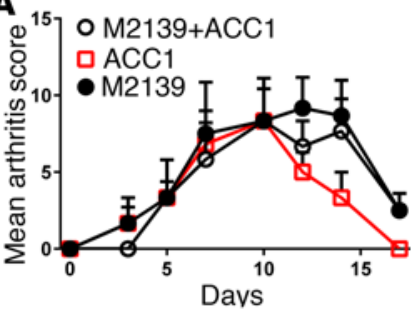

C
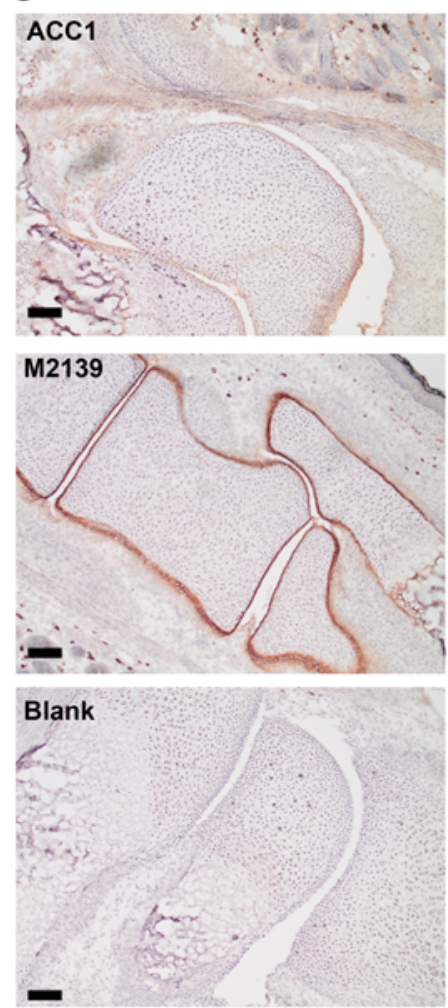

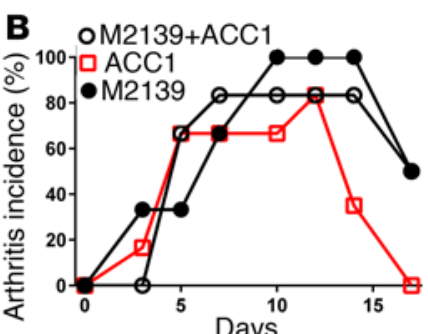

D
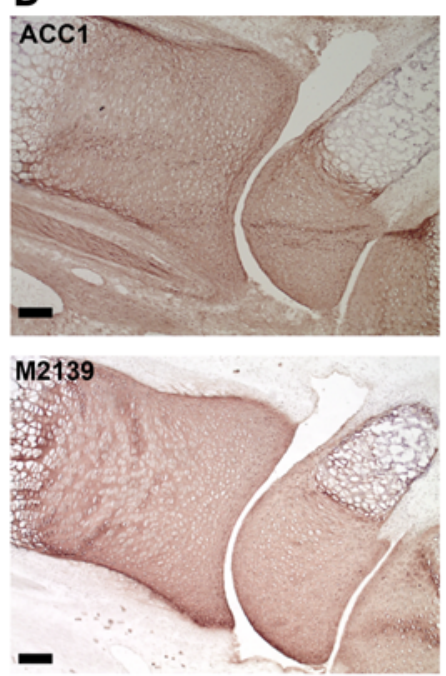

Blank

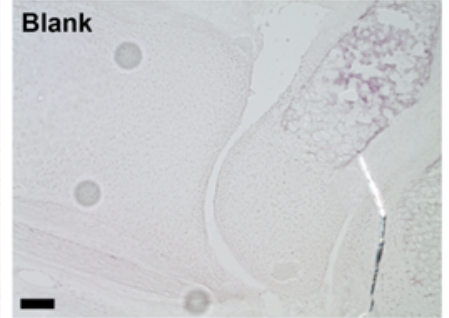

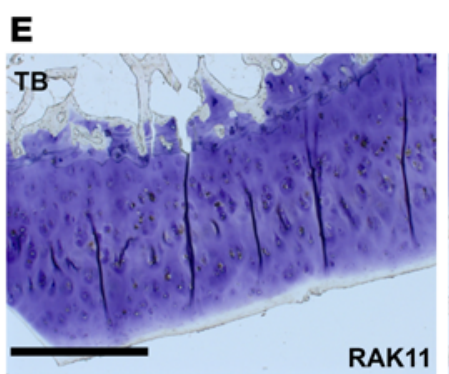
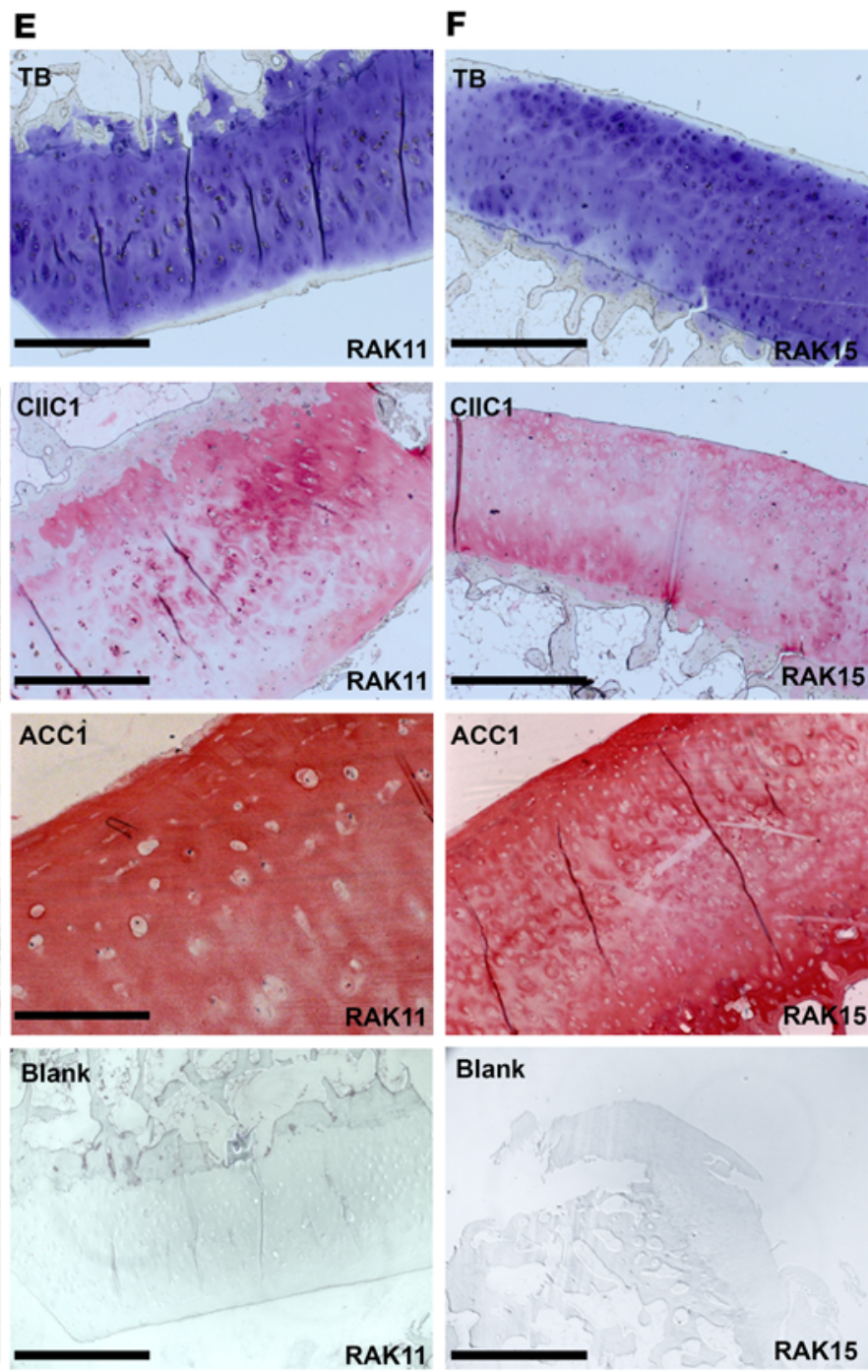

Blank

RAK15

Figure 4. Evaluation of ACC1 in mice and RA in terms of pathogenicity and cartilage-binding capacity. (A and B) ACC1-mediated arthritis in mice by passive transfer. Both arthritis score (A) and incidence (B) are shown. Four groups of B10.Q mice (6 mice in each group) were injected with PBS, ACC1, M2139, and a cocktail of M2139+ACC1, respectively. All mice received $25 \mu \mathrm{g}$ LPS from E. coli intraperitoneally on day 5 . Data represent mean \pm SEM ( $n=6$ in each group) in A. (C and $\mathbf{D}$ ) The cartilage binding of ACC1 to mouse cartilage was evaluated by immunohistochemical staining both in vivo (C) and in vitro (D). The knee joints and paws from adult mice were stained with $\mathrm{H} \& \mathrm{E}$ or toluidine blue. For in vivo staining, the knee joints from a 2-day-old neonate Cia9i mouse injected with biotinylated antibodies were used. To assess direct binding of mAbs to the tissue sections in vitro, limbs from 2-day-old naive Cia9i neonates were used. Scale bar: $100 \mu \mathrm{m}$ (C and $\mathbf{D})$. (E and $\mathbf{F})$ Immunohistochemical staining of cartilage from the finger joints of two rheumatoid arthritis (RA) patients. The deparaffinized sections $(5-8 \mu \mathrm{m})$ were treated with testicular hyaluronidase, followed by incubation with ACC1. The sections were visualized with permanent red (Dako) as color substrate. Nuclei were counterstained by hematoxylin. For positive control staining, deparaffinized sections were incubated with the mouse anti-CII mAb CIIC1 specific for triple-helical CII. Negative control staining lacked the primary anti-mouse IgC antibody. Samples were stained with ACC1, secondary antibody control (negative control), CIIC1 (positive control), and toluidine blue (TB). RAK11 and RAK15 represent two patients. Scale bar: 1,000 $\mu \mathrm{m}$ (E and F).

sulphate resulted in B-factors equivalent to those of surrounding Fab residues. The putative sulphate ion is well coordinated by amide groups of the motif arginine and surrounding heavy-chain residues (hW33, $\mathrm{hN} 53$, and hN56) as well as the carboxyl group of the peptide residue pE23 (E623 in CII) and thus contributes substantially to the Fab-peptide interface (Supplemental Figure 4). The strict conservation of an (unmodified) arginine, 3 positions before the motif threonine in all peptide sequences recognized by ACC1 may be partly explained by its importance for coordination and charge compensation of the counter ion, which in turn considerably contributes to interface formation.

The two glycines in the conserved peptide motif (RG-TG) play a crucial role in enabling the recognized epitope conformation: the first glycine adopts a backbone angle combination that is not accessible to other amino acids, and the second simply does not have a side chain that could cause steric clashes. 
Table 3. Data collection and refinement statistics

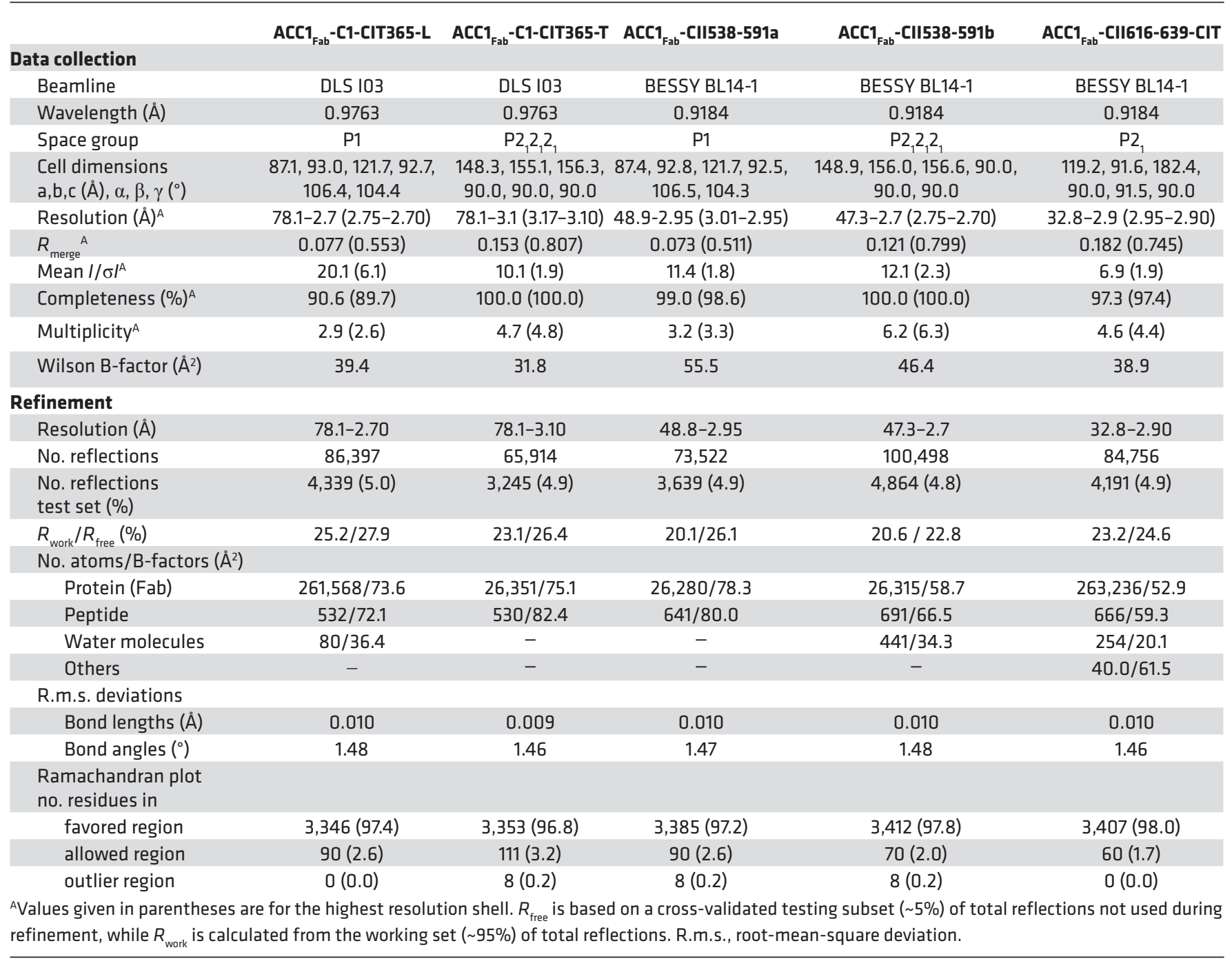

A sequence alignment showed that the RG-TG motif exists in all ACC1-binding epitopes (Supplemental Table 2). However, this motif is not unique to CII but is also found in almost all other types of collagen. To investigate ACC1 binding to this consensus sequence further, we set up an ELISA using immobilized heat-denatured collagens I, II, IX, and XI. No significant interaction of ACC1 with collagens I and IX could be detected, whereas clear signals were obtained for collagen II and XI (Supplemental Figure 7A). Both are cartilage-specific proteins, and they contain the same $\alpha$ chain, as CXI is a heterotrimeric collagen, with the $\alpha 3$ chain identical to the $\alpha 1$ chain in CII. Furthermore, the relative stability profiles for the binding sites on the recognized peptides are predicted to be lower than their neighboring regions (Supplemental Figure 7B), suggesting that ACC1 preferentially binds to RG-TG motifs located in structurally less stable regions on the triple helix.

Citrulline specificity of $C^{C I T 365}$ is governed by an arginine residue from ACC1. The C1 epitope, in this crystallographic study present in peptides C1-CIT365-L and C1-CIT365-T, is recognized by the ACC1 antibody only if the second arginine (R365) is modified to citrulline. Nevertheless, ACC1 does also recognize sequences that do not contain any citrulline, which indicates that it biases against the arginine, rather than for a citrulline. For instance, CII583-591 and CII616-639 both contain a proline at the equivalent position (Supplemental Table 3). In the $A C C 1_{\mathrm{Fab}}$-peptide complexes, the citrulline is relatively solvent exposed and makes side chain-specific hydrogen bonds with hR52 in only some of the complex copies per asymmetric unit (Supplemental Figure 5A). However, due to space constraints within the narrow paratope, the neutral citrulline side chain is always placed in proximity to the posi- 
A

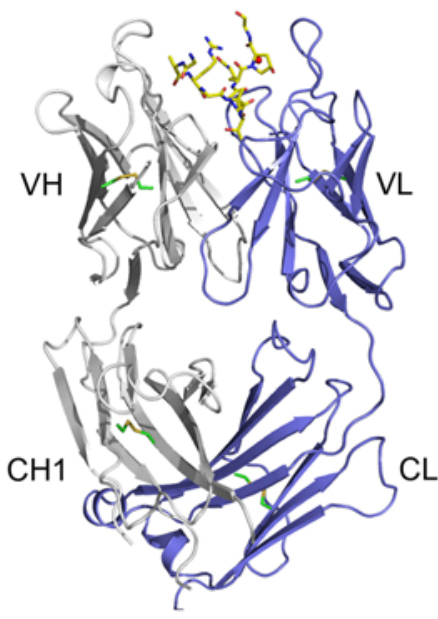

B

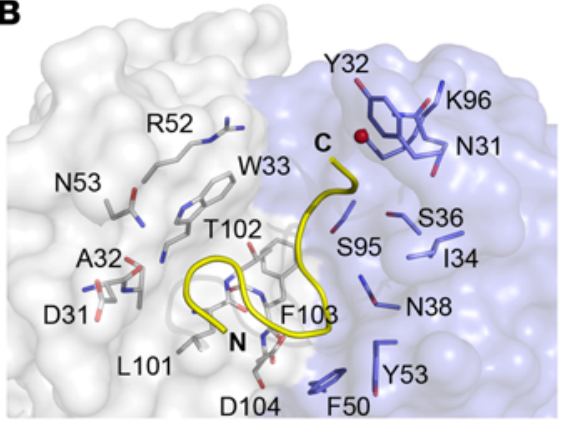

C

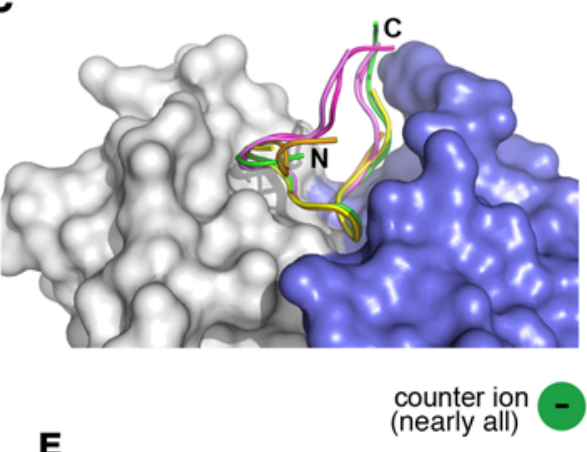

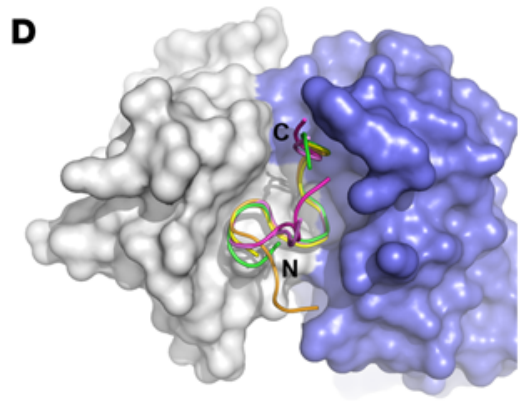

hD104-N
3. $0-3.4 \mathrm{~A}$ hF103-N

(34/40) $\quad 2.6-3.1 \mathrm{~A}$

$2.6-3.1 \mathrm{~A}$
$(39 / 40)$

$-081$

2.8-3.2 A $-0 \delta 1$

(all)
1 2.7-3.4A (12/40)

$\mathrm{Nn} 1$ or $\mathrm{Nn} 2$,

2.6-3.3 A (19/40)

4 2.7-3.4A (16/40)

6 2.8-3.4 A (32/40)

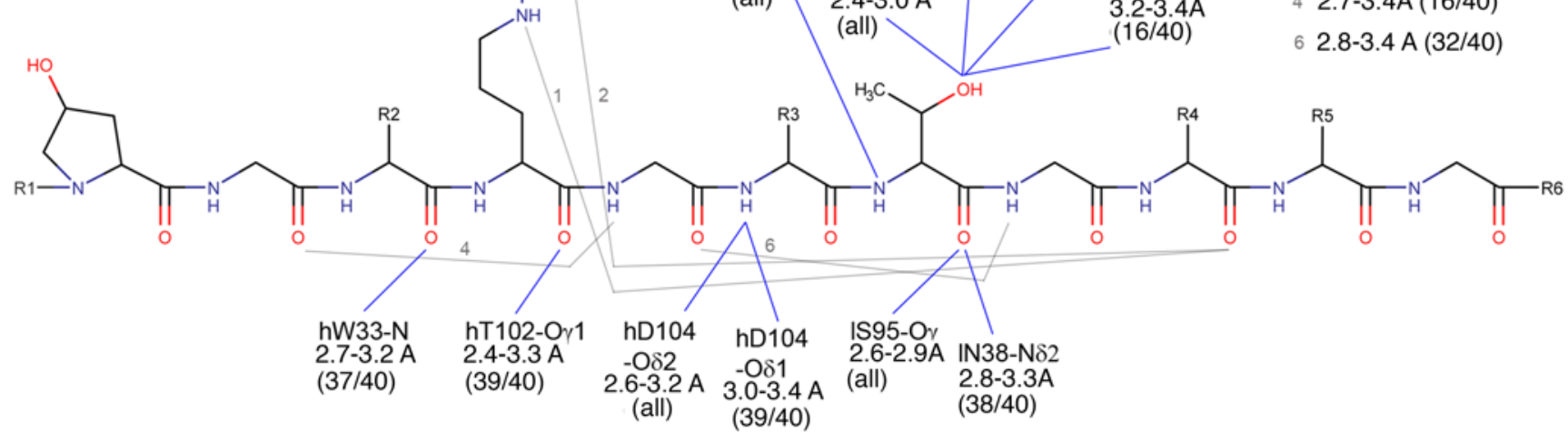

Figure 5. Crystal structures of the $\mathbf{A C C}_{\mathrm{Fab}}$-peptide complexes. (A) The $A C C 1_{\mathrm{Fab}}$ heavy and light chains are shown in white and blue, respectively. The bound

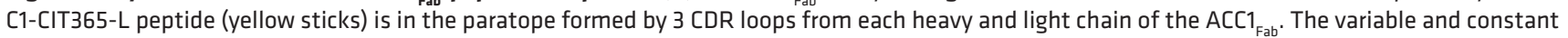
domains of both chains ( VH, VL, CH1, and CL) each contain a disulfide bond, shown as sticks with carbon atoms in green and sulfur atoms in yellow. (B) The dimensions of the paratope are outlined by the semitransparent molecular envelope of the ACC1 $_{\mathrm{Fab}}$, which is colored white for the heavy chain and blue for the light chain. The bound C1-CIT365-L peptide is depicted in yellow, and all ACC1 residues located within a 4-Å radius from the peptide are depicted as sticks with carbon atoms in white or light blue in accordance with heavy or light chain origin; oxygen atoms are depicted in red, and nitrogen atoms are depicted in blue. (C and $\mathbf{D}$ ) Comparison of the peptide-binding modes, with the paratopes viewed from the side (C) and the top (D). The 5 ACC1 ${ }_{\mathrm{Fab}}-$ peptide complexes were superimposed based on the heavy-chain variable domain. The molecular envelope of the $\mathrm{ACC1}_{\mathrm{Fab}}$ is colored as in $\mathbf{B}$, bound peptides are shown in yellow (C1-CIT365-L), orange (C1-CIT365-T), magenta (CII538-591 in the crystal of space group P2 2,2 $2_{1}$, mauve (CII538-591 in P1), and green (CII616$639-\mathrm{CIT})$. The orientation of the peptides is indicated by labeling of their $\mathrm{N}$ - and C-termini. (E) Schematic presentation of peptide-binding site in ACC1 ${ }_{\mathrm{Fab}}$. The nonconserved side chains (R2-R5) and invisible parts in electron density (R1, R6) are abbreviated. Only hydrogen bonds observed in at least a third of the 40 analyzed $\mathrm{ACC1}_{\mathrm{Fab}}$-peptide complexes are shown. Intrapeptide hydrogen bonds are indicated by black dotted lines, hydrogen bonds between the peptide and the Fab are indicated as solid blue lines. The heavy- or light-chain origin is indicated by an $\mathrm{h}$ or I suffix, respectively. The counter ion of unknown type is indicated by a green circle. CDR, complementarity-determining region; Fab, antigen-binding fragment; VH, heavy-chain variable domain; VL, light chain variable domain; $\mathrm{CH} 1$, heavy-chain constant domain; $\mathrm{CL}$, light-chain constant domain. 
A
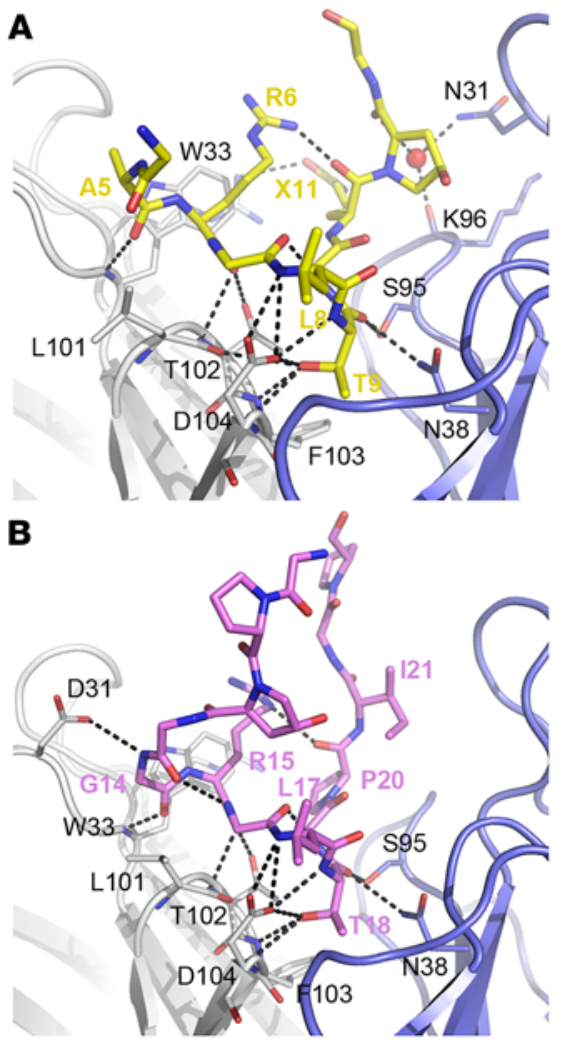

C

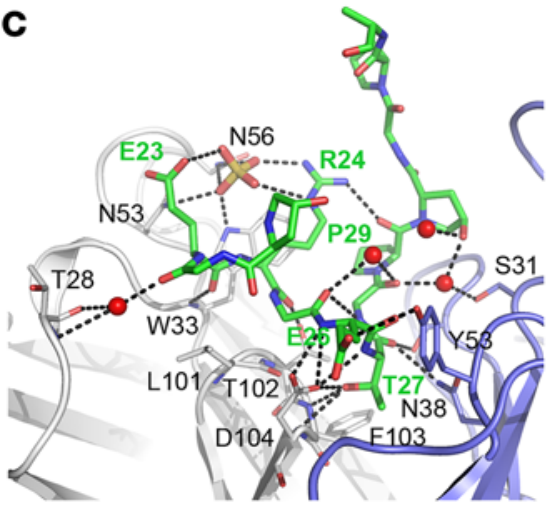

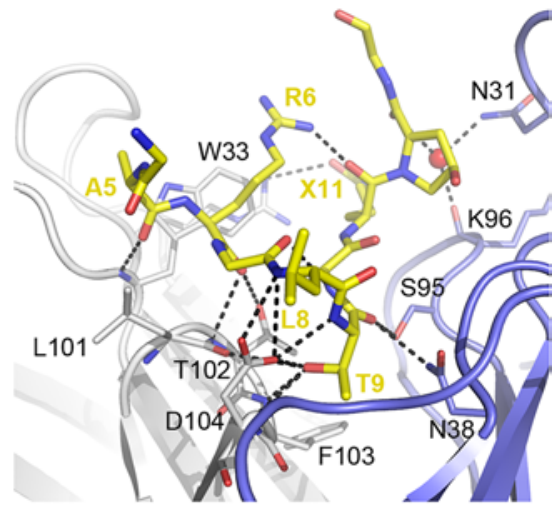
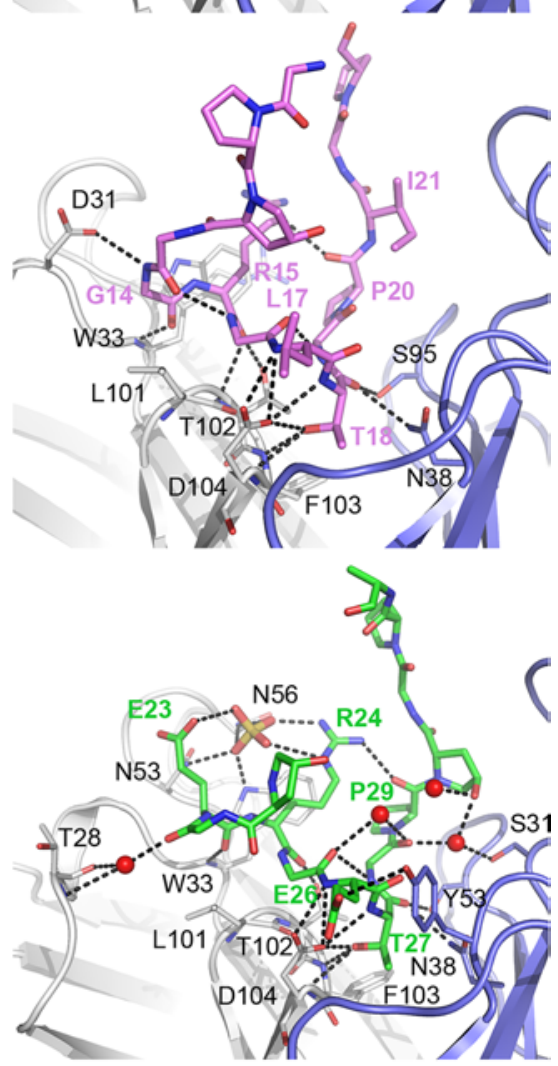

Figure 6. Hydrogen-bonding network formed upon peptide binding to ACC1, with emphasis on conserved interactions. The chosen orientation focuses on the conserved interactions between the threonine of the conserved peptide motif and the H3 CDR loop (see Supplemental Figure 5 for another orientation). The heavy and light chains of the $\mathrm{ACC1}_{\mathrm{Fab}}$ are shown in white and blue, respectively. Fab residues forming hydrogen bonds to the peptide are shown as sticks, with carbon atoms colored according to their chain of origin and labeled in black. To improve clarity, residues lying behind the peptide are not labeled. Hydrogen bonds are indicated as black dotted lines, water molecules are indicated as red spheres. The peptides are shown as thicker sticks, with carbon atoms in yellow (C1-CIT365-L), mauve (CII538-591 in space group p1), and green (CII616-639-CIT), respectively. All peptide residues other than glycine, proline (except the proline occurring in CII538-591 and Cll616-639-CIT at the same position as the citrulline in C1-CIT365-L

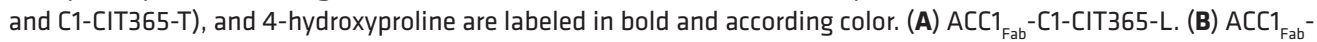
CII538-591 as in P1 space group. (C) ACC1 ${ }_{\mathrm{Fab}}$-CII616-639-CIT. (The hydrogen bonding networks of the complexes ACC1 Fab $^{-}$ C1-CIT365-T and ACC1 ${ }_{\mathrm{Fab}}$-CII538-591 in P2 2 2 2, are shown in Supplemental Figure 4). CDR, complementarity-determining region; Fab, antigen-binding fragment.

tively charged guanidinium group of hR52. Occurrence of an unmodified arginine at this peptide position would thus lead to an energetically unfavorable immediate neighborhood of two positive charges, which may explain the bias against such sequences by ACC1.

Comparison of CII epitope binding by other ACPAs and anti-CII antibodies. Three other crystal structures of Fab fragments recognizing CII epitopes are currently deposited in the Protein Data Bank. One is 
ACC4 (PDB:2W65), which specifically recognizes the C1 peptide with a citrulline at position 360, but which, in contrast to $\mathrm{ACC} 1$, does not bind the triple-helical form of the $\mathrm{C} 1$ epitope. We can thus compare how the same linear epitope is bound by ACC4 $\left(\mathrm{C}^{\mathrm{CIT360}}\right)$ and ACC1 $\left(\mathrm{C} 1^{\text {CIT365 }}\right)$ but with different modification states. The other two structures comprise the Fab-peptide complexes of the anti-CII antibodies CIIC1 (20) and M2139 (22), which recognize the native, i.e., triple-helical and unmodified, C1 and J1 epitopes of CII, respectively. Comparisons of these structures may allow identification of determinants of the conformational selectivity, i.e., single-chain versus triple-helical epitopes, of these antibodies.

The superposition of all $\mathrm{ACC}_{\mathrm{Fab}}$-peptide complexes shows an almost identical paratope conformation for the 3 distinct epitopes (Figure 7A). In contrast, the superimposition of $\mathrm{ACC}_{\mathrm{Fab}}-\mathrm{C} 1-365-\mathrm{L}$ ("Cit365") with ACC4 ${ }_{\mathrm{Fab}}-\mathrm{C} 1$ ("Cit360") revealed that the peptide-binding paratopes of both ACPAs do not coincide, despite the almost identical peptide sequences (Figure 7B). The binding site is shallower in the ACC4 $4_{\mathrm{Fab}}$ complex and shifted toward the heavy-chain CDR loops, which provide approximately $70 \%$ of the buried SASA (16), with the CDR L1 barely being involved and CDR L2 not being involved in peptide binding. This shallower binding site in ACC4 is primarily due to the extension of the H3 loop by 4 residues as compared with ACC1, while the shorter H3 loop confers ACC1 a deeper and more open-ended binding groove (Supplemental Figure 8, A and B), and an extension by 4 residues, as seen in ACC4, would fill the small cavity of the paratope that is especially crucial for peptide binding as it harbors the motif threonine.

The peptide conformation and orientation in the binding groove of $\mathrm{ACC1}_{\mathrm{Fab}}$ differs substantially from that observed for the $\mathrm{ACC}_{\mathrm{Fab}}$. The only conserved conformational feature is the type II $\beta$-turn at the peptide tip formed by the motif threonine and the preceding leucine. Furthermore, both the $\mathrm{C} 1$ epitope arginine (R365) and citrulline (Cit360) are recognized by ACC4 via a network of hydrogen bonds involving their side chain functional groups, while no such network involving the equivalent groups is formed upon binding of the alternatively modified (R360+Cit365) C1 epitope to ACC1. Thus, none of the C1 epitope-Fab interactions appear to be conserved between the two ACPAs.

Comparison of the $\mathrm{ACC1}_{\mathrm{Fab}}$ complex to the $\mathrm{CIIC}_{\mathrm{Fab}}$ and $\mathrm{M} 2139_{\mathrm{Fab}}$ complexes (Figure 7C and Supplemental Figure 8, C and D) revealed that the open-ended binding groove among these 3 triple-helical CII-binding antibodies is a shared feature. However, the paratopes of the latter two are wider due to an outward movement of CDRs $\mathrm{H} 2$ and L1; they are also shallower, as the $\mathrm{H} 3$ loop is again extended, by 7 and 4 residues, respectively. This enlarges the groove sufficiently to hold an intact triple-helical peptide and abolishes the deeper cavity that is crucial for peptide binding by ACC1, explaining why ACC1 can only bind to single $\alpha$ chain of CII epitope, most likely bulging out of a rigid triple-helical structure, as seen in the present crystal structures.

\section{Discussion}

There is a growing consensus that autoantibodies, RFs and APCAs, predict the development of RA, but their functional roles in initiating arthritis remain elusive. The present study suggests a mechanism on how an ACPA can induce arthritis. We demonstrated that a monoclonal ACPA, which is reactive with several wellknown citrulline peptide targets, also cross-reacts with triple-helical epitopes on joint cartilage-specific CII.

A single $\alpha$ chain needs a certain degree of flexibility within the triple-helical CII to ensure the mechanical resistance of collagen in joint cartilage. Citrullination may alter triple-helical stability if this modification disrupts favorable interchain and/or interhelical electrostatic interactions (23-27). The citrulline appears to be distinguished from its unmodified predecessor arginine via electrostatic-controlled interactions, which also allow cross-reactivity with other collagen epitopes containing fewer polar amino acids at the equivalent position. Thus, the antibody can specifically recognize several epitopes on CII or CXI molecules in joint cartilage besides the citrullinated peptides to which it binds in in vitro assays. The efficient and specific binding of ACC1 that exhibits typical features of an RA-associated ACPA to several epitopes exposed in cartilage in vivo causes proteoglycan depletion and an onset of a severe form of erosive joint inflammation.

So far, only antibodies that can specifically bind cartilage have been shown to induce arthritis. The best described ones are CII antibodies. Antibodies to many but not all CII triple-helical exposed epitopes are highly arthritogenic $(19,28)$. Antibodies to some other cartilage proteins, such as cartilage oligomeric matrix protein (29) and matrilin 1 (30), are clearly pathogenic as well. Importantly, a common feature of all known arthritogenic antibodies is that they can specifically bind cartilage in vivo (28). To our knowledge, RF or ACPA, representing the hallmark antibodies of RA, have thus far not been shown to transfer arthritis. There are, however, observations that render it rather likely that 
A
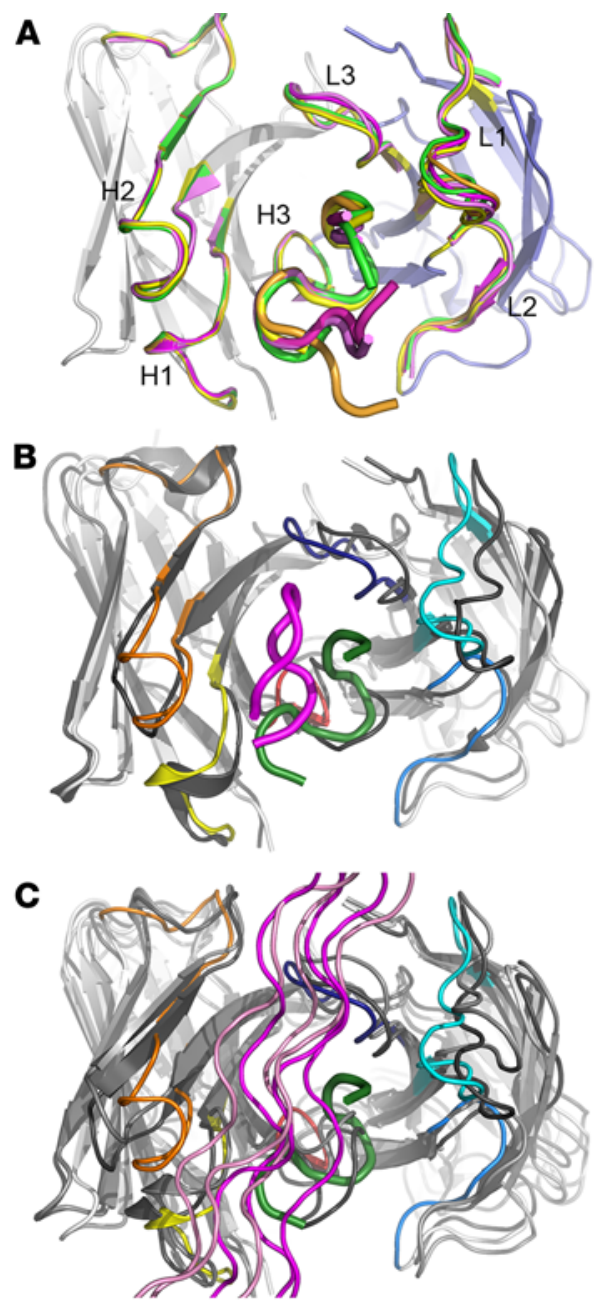
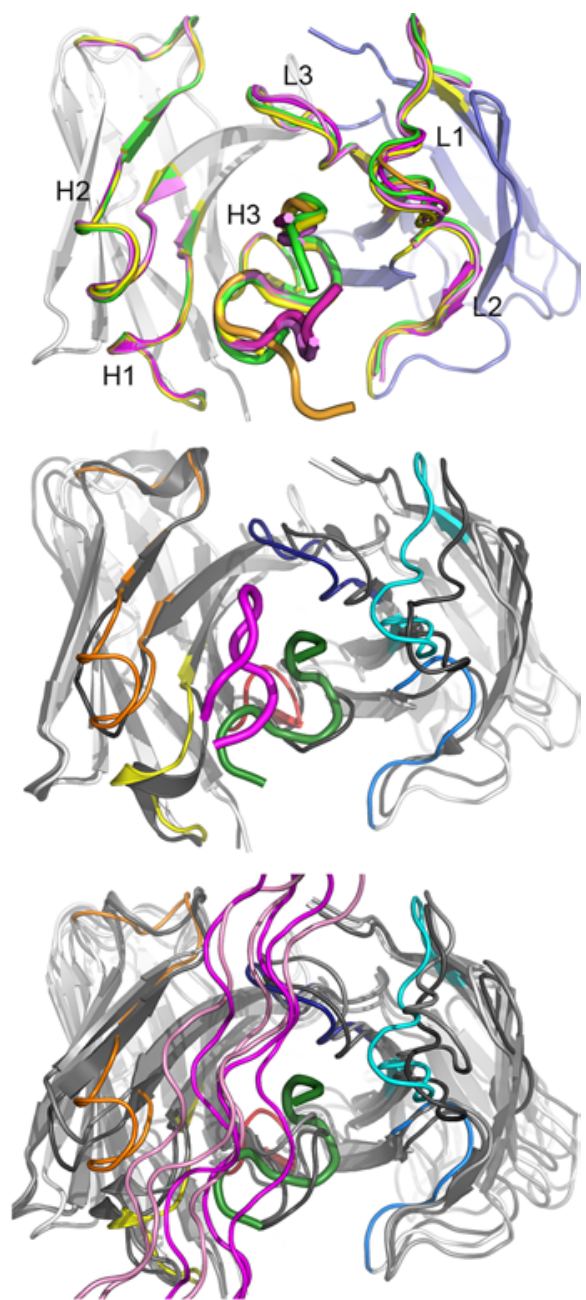

Figure 7. Comparison of CDR loop conformation and peptide binding by ACC1, ACC4, CIIC1, and M2139. (A) All 5 $A C C 1_{\text {Fab }}$ peptide complexes reported here were superimposed based on the $C \alpha$ atom coordinates of their respective $\mathrm{VH}$ domains. The backbone of the $\mathrm{ACC1}_{\mathrm{Fab}}$ in the complex with CII616-639-CIT is shown in stereo view, with heavy and light chains colored white and blue, respectively, except for the hypervariable CDR loop regions. The superimposed CDR loops and peptides of the respective $\mathrm{ACC1}_{\mathrm{Fab}}$-peptide complexes are shown in yellow for the C1-CIT365-L complex, bright orange for C1-CIT365-T, mauve for CII538-591 in space group P1, magenta for CII538-591 in space group P2, 2, 2, and green for the CII616-639-CIT complex. (B) Stereo view of the superimposed ACC1 ${ }_{\mathrm{Fab}}$-C1-CIT365-L (white) and ACC4 $\mathrm{Fab}_{\mathrm{F}}-\mathrm{C1Cit1}$ (gray) (PDB ID: 2W65) structures. The CDR loops of the ACC1 heavy chain are colored yellow (H1), orange $(\mathrm{H} 2)$, and red $(\mathrm{H} 3)$; those of the light chain are colored cyan (L1), marine (L2), and dark blue (L3). The cartoon representation of the C1-CIT365-L peptide bound to the $\mathrm{ACC}_{\mathrm{Fab}}$ is shown in dark green, and that of the C1Cit1 peptide bound

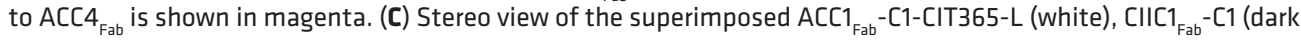
grey) (PDB ID: 2Y5T), and M2139 Fab $_{-11}$ (light gray) (PDB ID: 4BKL) structures. The CDR loops of the ACC1 heavy chain are colored as in B. The cartoon representation of the C1-CIT365-L peptide bound to the $\mathrm{ACC1}_{\mathrm{Fab}}$ is shown in dark green, and

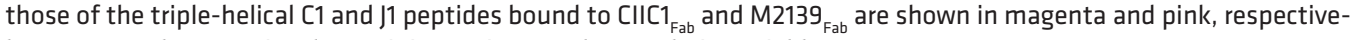
ly. CDR, complementarity-determining region; $\mathrm{VH}$, heavy-chain-variable.

RA autoantibodies contribute to the disease. It has recently been shown that healthy individuals with detectable ACPA titers, and most likely also RF, have detectable bone erosions (31). Such antibodies have also been shown to lack galactosylation of their Fc-attached glycans, rendering them more prone to $\mathrm{Fc}$ receptor interactions, which contribute to a variety of proinflammatory pathways, including activation of osteoclasts (11). Indeed, the contribution of both RFs and ACPAs to immune complexes enhances their stimulatory potential for macrophages (32). However, there are no conclusive data that these antibodies can specifically bind joint targets.

Antibodies specifically binding cartilage proteins like CII do occur in RA sera. However, only a small percentage of RA patients have antibodies specifically binding to triple-helical CII in vitro, as shown in 


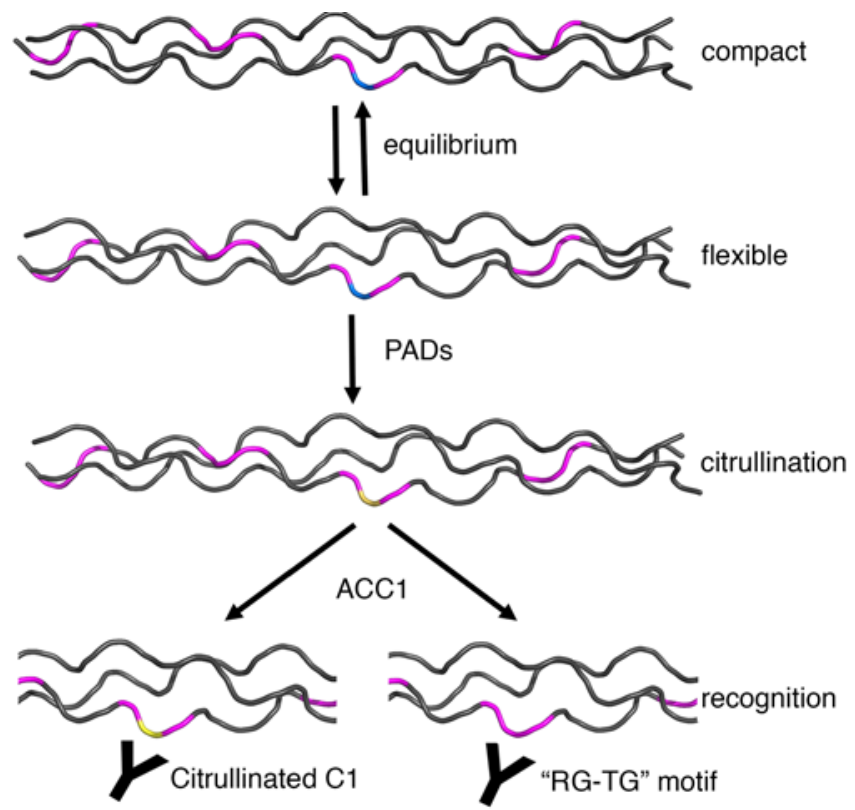

Figure 8. Pathogenicity of ACC1 mediated by cross-reactivity. Cll exists in a dynamic state composed of both compact and relatively flexible conformations, which is essential for ensuring the mechanical resistance of collagen in joint cartilage. Under inflammatory conditions, this equilibrium state of triple-helical structure is disrupted. The citrullination resulting from dysregulation of PADs on some arginine residues further deteriorates triple-helical stability, leading to exposure of Cll epitopes to immune systems. ACPAs, such as ACC1, attack CII via a shared structural motif, "RG-TG," within all the epitopes, including the ones bearing a citrulline residue (e.g., C1-CIT365), to which the recognition is governed by electrostatic potential-controlled citrulline specificity. The triple-helical CII is shown as gray ribbon with the motif RG-TC marked in magenta. The arginine residue within this motif is colored in blue, while the corresponding citrulline is in yellow. CII, collagen type II; PADs, peptidylarginine deiminases.

Figure 2. It is possible that antibodies that can bind cartilage in vivo occur more frequently than indicated by in vitro detection, as there are many cartilage proteins and many unique posttranslationally modified epitopes exposed. In addition, as shown here, exposure of epitopes on triple-helical CII might be restricted to states of destabilization, and such epitopes are not easily mimicked in vitro.

Interestingly, many ACPAs cross-react with citrullinated CII, and some of these antibodies bind to RA cartilage (14). The ACC1 antibody in the present study binds to classical citrullinated peptides (CCP2, CCP1) but also strongly binds to cartilage in vivo. However, from the present data, we cannot conclude which of the epitopes targeted by the antibodies are critical for the induction of arthritis. It is less likely that the citrullinated $\mathrm{C} 1$ epitope is the major target for initiating arthritis, since the arginine at position 365 of the $\mathrm{C} 1$ epitope is not readily citrullinated in vitro (14), although we cannot exclude that it could be citrullinated in vivo. Nevertheless, the ACC1 binding affinity to this epitope is lower than to other cross-reactive epitopes on CII, both unmodified and citrullinated. Hence, it is likely that the antibody can bind several epitopes in vivo. It is, however, very difficult to determine whether antibodies similar to ACC1 occur in RA sera due to the dynamic nature of the epitopes. In addition, a temporary interaction with a dynamic epitope might not efficiently induce tolerance in the developing B cells, whereas the antibodies, when produced by the matured plasma cells in larger quantities or trapped within immune complexes, could firmly bind to their targets.

Clearly, the molecular details of the recognition of a dynamically exposed epitope are of potential general relevance for the basic understanding of antibody-mediated autoimmune disease. It is of interest that a structurally conserved motif, "RG-TG," is shared among all ACC1-recognized CII antigens. The reactivity of ACPA is found to be governed by the conformational flexibility of the antigenic region and the amino acid residues surrounding the citrulline. The presence of a neutral amino acid with no or a small side chain (glycine and serine) on the $\mathrm{N}$-terminal site of the citrulline often favors recognition as compared with rigid residues (proline) at this position (33). In agreement with these observations, there is a glycine residue next to the citrulline in the "RG-TG(CIT)" motif preferred by ACC1. The CII region containing the RG-TG(CIT) motif has a low content of proline and 4-hydroxyproline (Figure 1A). Proline and 4-hydroxyproline stabilize the triple-helical structure, and a lack of these residues leads to a dynamically flexible and structurally distinct region of the protein (34).

Our structures show that all complexes share an almost identical peptide-binding mode, with three key determinants explaining the breadth of its cross-reactivity: (a) the conserved backbone interaction between the two glycines within the RG-TG motif, (b) the shape complementarity and multitude of polar interactions of the threonine side chain in the motif, and (c) the hydrogen bond network formed between the peptide backbone and the antibody. Furthermore, the citrulline specificity for $\mathrm{C}^{\mathrm{cit} 365}$ is governed by a posi- 
tively charged arginine residue placed near it in the binding site on ACC1. However, as this binding site feature makes the occurrence of positively charged residues energetically unfavorable, rather than specifically selecting for citrulline, other electrostatically neutral residues, such as proline and, possibly, also negatively charged residues, are accepted at this peptide position.

Several studies demonstrated that the $\mathrm{C} 1$ epitope of $\mathrm{CII}$ is frequently targeted in $\mathrm{RA}$. Although the reactivity for an unmodified $\mathrm{C} 1$ epitope seems to be restricted to a subgroup of RA patients, the citrullinated triple-helical form of $\mathrm{C} 1$ exhibited much higher prevalence among the targets. The two arginines of the $\mathrm{C} 1$ epitope, R360 and R365, potentially yield 3 neoepitopes upon citrullination, all of which can be targeted by antibodies from RA patients. Although a similar prevalence (approximately 30\%) of autoantibodies against the C1 epitope citrullinated at R360 (C1_CIT-R) and the one citrullinated at both R360 and R365 (C1_CIT-CIT) was observed in RA patients, the marked overlap (Figure $2 \mathrm{~B}$ ) between these two groups of autoantibodies strongly indicates the possible causative role of the citrulline at position 360 in autoreactivity toward the citrullinated $\mathrm{C} 1$ epitope. In contrast, approximately $10 \%$ of RA patients reacted to the unmodified $\mathrm{Cl}$ epitope or to the epitope with a citrulline at position 365 (C1_R-CIT). Thus, we hypothesize that R365 is probably converted to citrulline in vivo. On the other hand, citrullination of R360 is more frequent and can be detected in both human and mouse cartilage. Importantly, ACC1 could bind C1 epitope citrullinated at either position (R360 or R365) in ELISA, although it had higher affinity toward C1-CIT365 than C1-CIT360. In addition, ACC1 was also able to substantially affect the binding of RA autoantibodies with C1-CIT360 (the most frequent C1 epitope in RA) in a solid-phase competition assay. These data highlight the relevance of ACC1 reactivity in ACPA of human RA patients. Interestingly, the $\mathrm{mAb}$ specific for this epitope (ACC4) does not bind cartilage and cannot induce arthritis, but it can enhance the development of arthritis (16). However, ACC4 binds synovial cells, and it is possible that these antibodies are a part of immune complexes that could enhance the development of arthritis, as has been also shown in other experimental systems (35) and hypothesized to occur in RA (36).

In summary, we have demonstrated a molecular mechanism (Figure 8) that could explain how ACPAs that bind promiscuously in vitro to many peptides could induce arthritis in mice. Such antibodies bind cartilage specifically in vivo via cross-recognition of a flexible epitope on type II collagen, mimicking the citrulline-containing structure.

\section{Methods}

Patient population. In the present study, we used part of the TIRA2 cohort $(n=504)$, which enrolled patients with recent-onset RA between 2006 and 2009 (37). Symptom duration was at least 6 weeks but less than 12 months. At the time of serum sampling (i.e., baseline), no patient was treated with disease-modifying antirheumatic drugs. Healthy subjects from the Western Region Initiative to Gather Information on Atherosclerosis (WINGA) cohort $(n=284)$ served as controls.

Luminex immunoassay. Detection of autoantibody response using Luminex technology has been described previously $(38,39)$. Briefly, all biotinylated peptides were captured on beads via recognition of NeutrAvidin (Thermo Fischer Scientific), which was immobilized on the beads through amine coupling. Human serum samples were diluted 1:100 (v/v) in assay buffer (3\% BSA, 5\% milk powder, 0,1\% ProClin300, 0.05\% Tween 20, $100 \mu \mathrm{g} / \mathrm{ml}$ NeutrAvidin in PBS) and incubated for 60 minutes at room temperature on a shaker for preadsorption of unspecific antibodies. Then, the serum samples were transferred to a 384-well plate (Greiner Bio-One) containing peptide-coated beads by a liquid handler (CyBi-SELMA, CyBio). After incubation on a shaker for 75 minutes, all beads were washed with PBS-T (0.05\% Tween-20 in PBS) on a plate washer (EL406, Biotek) and resuspended in a solution containing the secondary anti-human IgG Fcy-PE (Jackson Immuno Research). After 40 minutes of incubation, the beads were washed with PBS-T, followed by measurement in a FlexMap3D (Luminex Corp.). The median fluorescence intensity (MFI) was used to quantify the interaction of serum antibody with given peptides.

Animals. For the study of collagen antibody-induced arthritis, the B10.Q mouse strain was used, while a $\mathrm{Cia} 9 \mathrm{i}$ congenic mouse strain was used for the immunohistochemical staining experiments. The congenic B10.Q (C57/B110.Q/rhd) mice originated from the Jan Klein mouse colony (Tubingen University, Tubingen, Germany) and were maintained by Rikard Holmdahl (rhd). Cia9i congenic strain is derived from B10.Q-NOD Cia9 congenic mice as previously described (40). All mice were kept and bred in a climate-controlled, specific pathogen-free environment (Federation of Laboratory Animal Science Associations II) in the animal house of the Division of Medical Inflammation Research, Karolinska Institutet and the Central Animal Laboratory of the University of Turku. All experiments were 
performed with 8- to 10-week-old male mice under standard conditions.

Passive transfer of collagen antibodies. Four groups of B10.Q mice were injected with PBS, ACC1, M2139, and a cocktail of M2139+ACC1, respectively. All mice received $25 \mu \mathrm{g}$ LPS from E. coli intraperitoneally on day 5 . The development of arthritis was monitored daily for a period of 12 days using an extended scoring protocol (19). Briefly, clinical arthritis is defined as swelling and redness in the joint; it was scored as follows: 1 point was given for each swollen or red toe; 1 point was given for each swollen joint (metatarsal phalangeal joints, metacarpal phalangeal joints, proximal interphalangeal joints, and distal interphalangeal joints); and 5 points were given for a swollen ankle. The maximum score per limb was 15, and the maximum score per mouse was 60 .

Histology. For histological assessment, knee joints and paws from adult mice were dissected, decalcified, dehydrated, and paraffin embedded as previously described (16). The procedure is detailed in the Supplemental Methods.

SPR determination. Binding of all synthetic triple-helical peptides to ACC1 was analyzed as previously described (22) by SPR using a Biacore T200 biosensor (GE Healthcare) at $25^{\circ} \mathrm{C}$. The procedure is detailed in the Supplemental Methods.

Immunohistochemical staining of $R A$ cartilage. Cartilage samples were fixed with $4 \%$ paraformaldehyde in PBS for 24 hours, decalcified with trichloroacetic acid, dehydrated, and embedded in paraffin. 5- to 8 - $\mu \mathrm{m}$-thick paraffin sections were cut and stained with H\&E and toluidine blue. Deparaffinized sections were treated with testicular hyaluronidase $\left(2 \mathrm{mg} / \mathrm{ml}\right.$ in PBS for 30 minutes at $37^{\circ} \mathrm{C}$ ) (Sigma-Aldrich). 3\% BSA in Tris-buffered saline was used for blocking. Then, $50 \mu \mathrm{g} / \mathrm{ml}$ ACC1 was added, incubated for 1 hour at room temperature, and visualized with a biotinylated rabbit anti-mouse antibody, alkaline phosphatase-conjugated streptavidin (Link and Label System; BioGenex), and permanent red (Dako) as color substrate. Nuclei were counterstained by hematoxylin. For positive control staining, deparaffinized sections were incubated with the mouse anti-CII mAb CIIC1 specific for triple-helical CII at $50 \mu \mathrm{g} / \mathrm{ml}$ for 1 hour at room temperature and detected as described above. Negative control staining lacked the primary anti-mouse IgG antibody.

ELISA. ELISAs were performed to analyze the interaction between ACC1 and different collagens (I, II, IX, and XI), as well as the binding of ACC1 to CCP-1 and control peptides. Details are provided in the Supplemental Methods. For solid-phase inhibition assay, the Pierce NeutrAvidin-Coated Plate (ThermoFisher Scientific) was first coated by $1 \mu \mathrm{g} / \mathrm{ml}$ C1-CIT360 or C1-CIT365 for 2 hours at room temperature, followed by incubation at room temperature for 2 hours with ACC1 at a concentration of $2 \mu \mathrm{g} / \mathrm{ml}$. A pool of RA sera diluted at 1:300 by Chonblock buffer (Chondrex Inc.) was added and incubated for 3 hours at room temperature. Then, the DELFIA measurement was performed as suggested by the manufacturer (Perkin Elmer) with secondary antibody DELFIA Eu-Labeled Anti-Human IgG to quantify the binding level of human autoantibodies.

Peptide design and synthesis. To explore the cross-reactivity of ACC1, a library of triple-helical peptides derived from human collagen (Supplemental Table 1) was synthesized by GL Biochem. In each peptide, the 24 amino acids of interest ( 8 triplets) are flanked by $5 \mathrm{~N}$-terminal and $5 \mathrm{C}$-terminal GPO repeats that help maintain its triple-helical conformation. Of the C-terminal 9 amino acids of interest, all but the last peptide overlap with the N-terminal 9 amino acids of the next peptide. Thus, each successive peptide sequence advances 15 amino acids along the triple-helical sequence of collagen II. Biotinylated CCP1 and its control arginine variant were synthesized by WuXi Apptech. For the peptides used in crystallization, 4 GPO repeats were added to the N-termini of C1-CIT365-L, C1-CIT365-T, and CII583-591. In addition, one GPO was added to the C-terminus of C1-CIT365-L, while 3 GPO repeats were added to the C-termini of C1-CIT365-T and CII583-591. For CII616-639, the peptide was synthesized by GL Biochem as described above. All other triple-helical peptides used in the present study were synthesized as covalently linked homotrimeric peptides with $5 \mathrm{~N}$-terminal and $5 \mathrm{C}$-terminal GPO repeats. In these peptides, two of the $\alpha$ chains are carboxy-terminally linked to the amino groups of two consecutive lysine residues added to the sequence of the third chain (41).

Triple-helical stability. The triple-helical stability was calculated via the "collagen stability calculator" (42).

Crystallization, data collection, and structure determination. Screening for crystallization conditions was performed in sitting-drop vapor diffusion mode at $293 \mathrm{~K}$ using PACT and PEGs sparse matrix screens (NeXtal, Qiagen), with $0.3-\mu 1$ drops pipetted by a robot being equilibrated against $60-\mu 1$ reservoirs. Crystals usually appeared under several conditions within 1-2 days. Details regarding the crystallization condition for each complex are provided in the Supplemental Methods. 
All crystals were flash frozen in liquid nitrogen prior to data collection at $100 \mathrm{~K}$ at the synchrotron beamlines listed in Table 3. Data were indexed and integrated on site with the respective autoprocessing pipelines XIA2-3d (43-48) or XDSapp (49) and scaled with AIMLESS from the CCP4 suite of programs (50). The crystals belong to space groups $\mathrm{P} 1, \mathrm{P} 2_{1}$, or $\mathrm{P} 2_{1} 2_{1} 2_{1}$, which always contained 8 copies of the $\mathrm{ACC}_{\mathrm{Fab}}$-peptide complex per asymmetric unit.

Initial phases were obtained by molecular replacement using preliminary data collected for the ACC1+C1-CIT365-L complex (for which only low-occupancy peptide binding was observed), the program PHASER (51), and crystal structures of the heavy and light chain constant domain, and variable domain dimers of the mouse anti-c-myc antibody 9E10 Fab fragment (PDB code 2OR9, ref. 52) were used as search models. Gradually improving models of the $\mathrm{ACC} 1_{\mathrm{Fab}}$ were then used to solve all subsequent $\mathrm{ACC1}_{\mathrm{Fab}}$ complex structures by molecular replacement. Manual model building using COOT (53) was alternated with TLS and restrained refinement in REFMAC5 (54). A set of 4.9\%-5\% randomly selected reflections was used to monitor $\mathrm{R}_{\text {free }}$. Water molecules were added in COOT for some of the complexes. Data collection and refinement statistics are provided in Table 3 . Most of the heavy-chain and light-chain copies of the final models contain residues $1-218$, but several of them are either shorter (at $\mathrm{N}$ - or C-terminus) or extended (at the $\mathrm{C}$-terminal end) by 1-2 residues, depending on the visibility of the termini in the electron density map. Furthermore, residues 132-136 of the heavy chain are often poorly resolved and thus not always modeled. The observed electron density for the peptide varies by a few residues from the 8 copies present in the asymmetric unit for each of the respective complexes (the maximal extent is indicated in Table 2 by underlining). In several of the ACC $1_{\mathrm{Fab}}$-peptide complex structures $2-3$ of the Fab copies show poorer electron density, considerably higher B-factors, and larger backbone RMSD deviations than the remaining copies, most likely indicating poorer packing in the crystal lattice. A higher peak of electron density near the guanidinium group of the conserved peptide arginine residue indicates the presence of a counter ion, which could, however, not be identified and was thus not modeled, aside from the ACC $1_{\mathrm{Fab}}$-CII616-639-CIT complex. All models had good stereochemistry, with $>97 \%$ of the residues in the most favored regions of the Ramachandran plot (Table 3). Molecular surfaces were analyzed with the Protein Interfaces, Surfaces, and Assemblies Service at the European Bioinformatics Institute (55), and elbow angles were analyzed with RBOW (56). Least-square fitting, as implemented in COOT, was used for structure comparisons and RMSD calculations (53). Figures were prepared with PyMol (57). The crystallographic coordinates and structure factors of the $\mathrm{ACC}_{\mathrm{Fab}}$-peptide complexes have been deposited in the Protein Data Bank with the accession codes 5MU0 (C1-CIT365-L), 5MUB (C1-CIT365-T), 5MU2 (CII583591 in $\mathrm{P} 2{ }_{1} 2_{1} 2_{1}$ ), $5 \mathrm{MV} 3$ (CII583-591 in P1), and 5MV4 (CII616-639-CIT).

Statistics. For the arthritis experiments, quantitative data are expressed as mean \pm SEM. Arthritis incidence was analyzed using Fisher's exact test, and comparison of severity was performed using the nonparametric Mann-Whitney $U$ test. Differences with $P$ values of less than 0.05 were considered statistically significant. $\mathrm{R}$ and various $\mathrm{R}$ packages were used for data analysis and statistics for Luminex data (58). The nonparametric Wilcoxon rank-sum test was applied for statistical analysis of differences in MFI values obtained for comparisons of RA patients and healthy controls. Reactivity of a peptide was considered positive if the MFI value was above an MFI threshold set as the median plus $5 \times$ MAD (median absolute deviation) of the MFI values obtained for the healthy control.

Study approval. Informed consent was obtained from patients and healthy controls, and the study protocol was approved by the regional ethics review board in Linköping. Human cartilage samples were obtained from RA patients experiencing synovectomy, knee replacement, or finger joint replacement. Ethics approval was obtained from the institutional review board at University Hospital Frankfurt am Main, and informed consent was also obtained from all patients. The animal experiments were approved by the Stockholm Ethical Committee (N490/12, H2014-483) and the national Animal Experiment Board in Finland (ESAVI/439/04.10.07/2017).

\section{Author contributions}

$\mathrm{RH}$ and CG conceived and designed experiments; CG, DD, DT, BL, EL, HB, NS, CH, BA, and PN performed experiments and analyzed data; TS and AK collected the TIRA2 RA cohort samples; JV, JK, RS, and GBF synthesized the triple-helical peptides; and CG, DT, DD, HB, and RH wrote the paper. 


\section{Acknowledgments}

This study was supported by the Swedish Strategic Science Foundation (RB13-015), the Knut and Alice Wallenberg Foundation (KAW 2010.0148), the Swedish Research Council (2015-02662), and the EU Innovative Medicine initiative BeTheCure grant (IMI 115142). We thank Carlos and Kristina Palestro for taking excellent care of the experimental animals. We would also like to thank the GE Healthcare DemoLab at the Science for Life Laboratory Stockholm for technical support with the SPR assays. We further acknowledge the Protein Science Facility at the Karolinska Institutet for providing crystallization infrastructure and access to the circular dichroism machine. We thank Bernhard Lohkamp and Dominic Böth for assistance with crystallographic data collections. We would especially like to thank all the patients who donated samples for this study. We thankfully acknowledge access to beamlines at the Diamond Light Source, the Berliner Elektronenspeicherring-Gesellschaft für Synchrotronstrahlung (BESSY), and the European Synchrotron Radiation Facility (proposals mx8492, mx1551, mx1639) as well as their staff for assistance with crystal testing and data collection. The research leading to these results has received funding from the European Community's Seventh Framework Programme (FP7/2007-2013) under BioStruct-X (grant agreement 283570). HB received support from the German Federal Ministry of Education and Research ArthroMark (project 4, 01 EC 1009C) and the Federal State of Hesse (LOEWE-project: IME Fraunhofer Project Group TMP at Goethe University).

Address correspondence to: Rikard Holmdahl, Karolinska Institute, Department of Medical Biochemistry and Biophysics, Medical Inflammation Research, Scheeles väg 2, SE-171 77 Stockholm, Sweden. Phone: 46.08.524.84607; Email: Rikard.Holmdahl@ki.se.

1. McInnes IB, Schett G. The pathogenesis of rheumatoid arthritis. N Engl J Med. 2011;365(23):2205-2219.

2. Sangha O. Epidemiology of rheumatic diseases. Rheumatology (Oxford). 2000;39 Suppl 2:3-12.

3. Schellekens GA, et al. The diagnostic properties of rheumatoid arthritis antibodies recognizing a cyclic citrullinated peptide. Arthritis Rheum. 2000;43(1):155-163.

4. Aho K, Heliovaara M, Maatela J, Tuomi T, Palosuo T. Rheumatoid factors antedating clinical rheumatoid arthritis. J Rheuma tol. 1991;18(9):1282-1284.

5. Rantapaa-Dahlqvist S, et al. Antibodies against cyclic citrullinated peptide and IgA rheumatoid factor predict the development of rheumatoid arthritis. Arthritis Rheum. 2003;48(10):2741-2749.

6. Burska AN, et al. Autoantibodies to posttranslational modifications in rheumatoid arthritis. Mediators Inflamm. 2014;2014:492873.

7. Law SC, et al. T-cell autoreactivity to citrullinated autoantigenic peptides in rheumatoid arthritis patients carrying HLA-DRB1 shared epitope alleles. Arthritis Res Ther. 2012;14(3):R118.

8. Sokolove J, Zhao XY, Chandra PE, Robinson WH. Immune complexes containing citrullinated fibrinogen costimulate macrophages via Toll-like receptor 4 and fc gamma receptor. Arthritis Rheum-Us. 2011;63(1):53-62.

9. Trouw LA, et al. Anti-cyclic citrullinated peptide antibodies from rheumatoid arthritis patients activate complement via both the classical and alternative pathways. Arthritis Rheum-Us. 2009;60(7):1923-1931.

10. Harre U, et al. Induction of osteoclastogenesis and bone loss by human autoantibodies against citrullinated vimentin. J Clin Invest. 2012;122(5):1791-1802.

11. Harre U, et al. Glycosylation of immunoglobulin G determines osteoclast differentiation and bone loss. Nat Commun. $2015 ; 6: 6651$.

12. Chatzidionysiou K, et al. Highest clinical effectiveness of rituximab in autoantibody-positive patients with rheumatoid arthritis and in those for whom no more than one previous TNF antagonist has failed: pooled data from 10 European registries. Ann Rheum Dis. 2011;70(9):1575-1580.

13. Rombouts Y, et al. Anti-citrullinated protein antibodies acquire a pro-inflammatory Fc glycosylation phenotype prior to the onset of rheumatoid arthritis. Ann Rheum Dis. 2015;74(1):234-241.

14. Haag S, et al. Identification of new citrulline-specific autoantibodies, which bind to human arthritic cartilage, by mass spectrometric analysis of citrullinated type ii collagen. Arthritis Rheumatol. 2014;66(6):1440-1449.

15. Holmdahl R, Malmstrom V, Burkhardt H. Autoimmune priming, tissue attack and chronic inflammation The three stages of rheumatoid arthritis. Eur J Immunol. 2014;44(6):1593-1599.

16. Uysal H, et al. Structure and pathogenicity of antibodies specific for citrullinated collagen type II in experimental arthritis. $J$ Exp Med. 2009;206(2):449-462.

17. Burkhardt H, Sehnert B, Bockermann R, Engstrom A, Kalden JR, Holmdahl R. Humoral immune response to citrullinated collagen type II determinants in early rheumatoid arthritis. Eur J Immunol. 2005;35(5):1643-1652.

18. Ricard-Blum S, Ruggiero F. The collagen superfamily: from the extracellular matrix to the cell membrane. Pathol Biol. 2005;53(7):430-442.

19. Nandakumar KS, Svensson L, Holmdahl R. Collagen type II-specific monoclonal antibody-induced arthritis in mice: description of the disease and the influence of age, sex, and genes. Am J Pathol. 2003;163(5):1827-1837.

20. Dobritzsch D, et al. Crystal structure of an arthritogenic anticollagen immune complex. Arthritis Rheum. 2011;63(12):3740-3748.

21. Lindh I, et al. Type II collagen antibody response is enriched in the synovial fluid of rheumatoid joints and directed to the same 
major epitopes as in collagen induced arthritis in primates and mice. Arthritis Res Ther. 2014;16(4):R143.

22. Raposo B, et al. Epitope-specific antibody response is controlled by immunoglobulin $\mathrm{V}(\mathrm{H})$ polymorphisms. J Exp Med. 2014;211(3):405-411

23. Parmar AS, James JK, Grisham DR, Pike DH, Nanda V. Dissecting electrostatic contributions to folding and self-assembly using designed multicomponent peptide systems. J Am Chem Soc. 2016;138(13):4362-4367.

24. Fallas JA, Dong J, Tao YJ, Hartgerink JD. Structural insights into charge pair interactions in triple helical collagen-like proteins J Biol Chem. 2012;287(11):8039-8047.

25. Fallas JA, Gauba V, Hartgerink JD. Solution structure of an ABC collagen heterotrimer reveals a single-register helix stabilized by electrostatic interactions. J Biol Chem. 2009;284(39):26851-26859.

26. Berisio R, De Simone A, Ruggiero A, Improta R, Vitagliano L. Role of side chains in collagen triple helix stabilization and partner recognition. J Pept Sci. 2009;15(3):131-140.

27. Yang W, Chan VC, Kirkpatrick A, Ramshaw JA, Brodsky B. Gly-Pro-Arg confers stability similar to Gly-Pro-Hyp in the collagen triple-helix of host-guest peptides. J Biol Chem. 1997;272(46):28837-28840.

28. Nandakumar KS, Holmdahl R. Antibody-induced arthritis: disease mechanisms and genes involved at the effector phase of arthritis. Arthritis Res Ther. 2006;8(6):223.

29. Geng H, Nandakumar KS, Pramhed A, Aspberg A, Mattsson R, Holmdahl R. Cartilage oligomeric matrix protein specific antibodies are pathogenic. Arthritis Res Ther. 2012;14(4):R191.

30. Hansson AS, Johannesson M, Svensson L, Nandakumar KS, Heinegard D, Holmdahl R. Relapsing polychondritis, induced in mice with matrilin 1, is an antibody- and complement-dependent disease. Am J Pathol. 2004;164(3):959-966.

31. Hecht C, et al. Additive effect of anti-citrullinated protein antibodies and rheumatoid factor on bone erosions in patients with RA. Ann Rheum Dis. 2015;74(12):2151-2156.

32. Laurent L, et al. IgM rheumatoid factor amplifies the inflammatory response of macrophages induced by the rheumatoid arthritis-specific immune complexes containing anticitrullinated protein antibodies. Ann Rheum Dis. 2015;74(7):1425-1431.

33. Trier NH, Leth ML, Hansen PR, Houen G. Cross-reactivity of a human IgG(1) anticitrullinated fibrinogen monoclonal antibody to a citrullinated profilaggrin peptide. Protein Sci. 2012;21(12):1929-1941.

34. Fields GB. Synthesis and biological applications of collagen-model triple-helical peptides. Org Biomol Chem. 2010;8(6):1237-1258

35. Kuhn KA, et al. Antibodies against citrullinated proteins enhance tissue injury in experimental autoimmune arthritis. JClin Invest. 2006;116(4):961-973.

36. Catrina AI, Svensson CI, Malmström V, Schett G, Klareskog L. Mechanisms leading from systemic autoimmunity to joint-specific disease in rheumatoid arthritis. Nat Rev Rheumatol. 2017;13(2):79-86.

37. Svard A, et al. Associations with smoking and shared epitope differ between IgA- and IgG-class antibodies to cyclic citrullinated peptides in early rheumatoid arthritis. Arthritis Rheumatol. 2015;67(8):2032-2037.

38. Ayoglu B, et al. Autoantibody profiling in multiple sclerosis using arrays of human protein fragments. Mol Cell Proteomics. 2013;12(9):2657-2672.

39. Ayoglu B, et al. Bead arrays for antibody and complement profiling reveal joint contribution of antibody isotypes to C3 deposition. PLoS One. 2014;9(5):e96403.

40. Lindqvist AK, Johannesson M, Johansson AC, Nandakumar KS, Blom AM, Holmdahl R. Backcross and partial advanced intercross analysis of nonobese diabetic gene-mediated effects on collagen-induced arthritis reveals an interactive effect by two major loci. J Immunol. 2006;177(6):3952-3959.

41. Fields CG, Lovdahl CM, Miles AJ, Hagen VL, Fields GB. Solid-phase synthesis and stability of triple-helical peptides incorporating native collagen sequences. Biopolymers. 1993;33(11):1695-1707.

42. Persikov AV, Ramshaw JA, Brodsky B. Prediction of collagen stability from amino acid sequence. J Biol Chem 2005;280(19):19343-19349.

43. Collaborative Computational Project N. The CCP4 suite: programs for protein crystallography. Acta Crystallogr D Biol Crystallogr. 1994;50(Pt 5):760-763.

44. Sauter NK, Grosse-Kunstleve RW, Adams PD. Robust indexing for automatic data collection. J Appl Crystallogr. 2004;37(Pt 3):399-409.

45. Winter G, Lobley CM, Prince SM. Decision making in xia2. Acta Crystallogr D Biol Crystallogr. 2013;69(Pt 7):1260-1273

46. Evans P. Scaling and assessment of data quality. Acta Crystallogr D Biol Crystallogr. 2006;62(Pt 1):72-82.

47. Zhang Z, Sauter NK, van den Bedem H, Snell G, Deacon AM. Automated diffraction image analysis and spot searching for high-throughput crystal screening. J Appl Crystallogr. 2006;39:112-119.

48. Kabsch W. Xds. Acta Crystallogr D Biol Crystallogr. 2010;66(Pt 2):125-132.

49. Sparta KM, Krug M, Heinemann U, Mueller U, Weiss MS. Xdsapp2.0. J Appl Crystallogr. 2016;49:1085-1092

50. Evans PR, Murshudov GN. How good are my data and what is the resolution? Acta Crystallogr D. 2013;69(Pt 7):1204-1214.

51. McCoy AJ, Grosse-Kunstleve RW, Adams PD, Winn MD, Storoni LC, Read RJ. Phaser crystallographic software. J Appl Crystallogr. 2007;40(Pt 4):658-674

52. Krauss N, et al. The structure of the anti-c-myc antibody 9E10 Fab fragment/epitope peptide complex reveals a novel binding mode dominated by the heavy chain hypervariable loops. Proteins. 2008;73(3):552-565

53. Emsley P, Lohkamp B, Scott WG, Cowtan K. Features and development of Coot. Acta Crystallogr D Biol Crystallogr. 2010;66(Pt 4):486-501.

54. Murshudov GN, Vagin AA, Dodson EJ. Refinement of macromolecular structures by the maximum-likelihood method. Acta Crystallogr D Biol Crystallogr. 1997;53(Pt 3):240-255.

55. Krissinel E, Henrick K. Inference of macromolecular assemblies from crystalline state. J Mol Biol. 2007;372(3):774-797.

56. Stanfield RL, Zemla A, Wilson IA, Rupp B. Antibody elbow angles are influenced by their light chain class. J Mol Biol. 2006;357(5):1566-1574

57. The PyMOL Molecular Graphics System, Version 1.8 Schrödinger, LLC. https://www.pymol.org/. Accessed June $20,2017$.

58. R Core Team (2017). R: A language and environment for statistical computing. R Foundation for Statistical Computing, Vienna, Austria. http://www.R-project.org/. Accessed June 20, 2017. 